\title{
Interval Estimation of Motion Intensity Variation using the Improved Inception-V3 Model
}

\author{
Wendong Wang ( $\nabla$ wdwang@nwpu.edu.cn ) \\ Northwestern Polytechnical University \\ Hanhao Li \\ Northwestern Polytechnical University \\ Chengzhi Zhao \\ Northwestern Polytechnical University \\ Dezhi Kong \\ Northwestern Polytechnical University \\ Peng Zhang \\ Northwestern Polytechnical University
}

\section{Research Article}

Keywords: Improved inception-v3 model, Motion intensity variation interval estimation, DQN-based trajectory optimization

Posted Date: March 24th, 2021

DOI: https://doi.org/10.21203/rs.3.rs-335444/v1

License: (c) (i) This work is licensed under a Creative Commons Attribution 4.0 International License. Read Full License 


\title{
Interval Estimation of Motion Intensity Variation using the improved Inception-V3 model
}

\author{
Wendong Wang ${ }^{1 *}$, Hanhao Li $^{1}$, Chengzhi Zhao ${ }^{1}$, Dezhi Kong ${ }^{1}$ and Peng Zhang ${ }^{2}$ \\ ${ }^{1}$ School of Mechanical Engineering, Northwestern Polytechnical University, Xi'an 710072 China \\ ${ }^{2}$ Training Center for Engineering Practices, Northwestern Polytechnical University, Xi' an 710072 China \\ E-mail: wdwang@nwpu.edu.cn
}

\begin{abstract}
In the process of acute resistance exercise, repeated variation in motion intensity can lead to muscle fatigue and heart failure. Therefore, acquiring the interval of motion intensity variation in time the training pattern and effect can be improved by acquiring the interval of motion intensity variation. In order to achieve this goal, an improved Inception-V3 model is proposed for motion intensity variation interval estimation. The MIVIE(Motion Intensity Variation Interval Estimation) dataset consisting of Strong, Moderate, Weak groups achieve centralized and uninterrupted collection. Then, the multi-modal fusion vectors of time-frequency eigenvalues are stacked up to $227 \times 227$ grayscale images fed into improved inception-CNN. Finally, the manipulator's trajectory optimization is completed under the guidance of ATO-DQN (Adaptive Trajectory Optimization-Deep Q Network) algorithm based on the motion intensity interval estimation. This work can improve the non-stationary effect of motor speed caused by changes in motion intensity during rehabilitation, which can better guarantee the safety of patients.
\end{abstract}

Keywords: Improved inception-v3 model, Motion intensity variation interval estimation, DQN-based trajectory optimization.

\section{Introduction}

With the development of medical technology, many more diseases can be cured. However, for patients with hemiplegia, the current main medical treatments are still nursing and rehabilitation training[1]. Through targeted treatments that depend on the appropriate diagnosis and therapy measures, the movement ability of patients can be partially and even completely restored[2]. Thus, rehabilitation training with the assistance of an exoskeleton arm is proposed to alleviate this practical problem[3]. Patients can gain the ability to conduct rehabilitation exercises independently through the exoskeleton rehabilitation robot, which will reduce the treatment cost and obtain more effective treatment[4]. In the process of acute resistance exercise, repeated variation in motion intensity can lead to muscle fatigue and heart failure. In order to optimize the training mode and improve the training effect, the reasonable use of this information can effectively avoid the motor out-of-step in the trajectory control of the manipulator[5].

The existing method[6, 7], which uses the heart rate as a detection mechanism, has been mainly used in medical diagnosis. Compared with EMG signals or EEG signals, the heart rate could alleviate individual differences and would be more easily detected by wearable devices[8, 9]. In terms of the existing research results[10,11], collecting accurate feedback signals and developing accurate and effective control strategies could improve the efficiency and quality of rehabilitation training.

Combined with medical and kinesiology theories, motion intensity refers to the degree of force exerted by the human body when performing actions and the degree of tension of the body. The motion intensity directly affects the stimulation effect of the current motion on the human body[12]. Existing research has put forward the algorithms and framework for heart rate monitoring during intensive exercise[13, 14]. Given the strong feature learning capabilities of CNNs, the researchers have been active in applying deep neural networks to solve the existing time-series classification problems. In the literature[15-17], the researchers used conditional and gated restricted Boltzmann machines, deep belief networks, recurrent neural networks, auto encoders, HMMs, and also extended form of $\mathrm{CNN}$ to improve the details of various problems.

Specifically, the technical contributions of this paper can be concluded as follows: MIVIE dataset consisting of Strong, Moderate, Weak groups realizes the data collection of setting specific situation according to the individual's condition. We build an improved Inception-V3 model is proposed for motion intensity variation interval estimation. the manipulator's trajectory optimization is completed under the guidance of ATO-DQN algorithm based on the motion intensity interval estimation. In the field of technological innovation, use physiological information obtained by BMD101 ECG sensor and angular acceleration sensor to comprehensively judge and divide the boundary of rehabilitation motion intensity through the improved model achieve complementary advantages and mitigate the negative effects.

\section{Related Work}

The difference of rehabilitation motion intensity results in the difference of rehabilitation effect for various diseases among different populations. Weak and moderate intensity motion is associated with protection against chronic diseases, especially cardiovascular disease[18, 19]. High intensity interval training (HIIT) may be an incredibly effective way to increase $\mathrm{VO}_{2 \text { peak }}$ and improve cardiorespiratory fitness compared to moderate intensity continuous training[18]. Therefore, the setting of rehabilitation motion intensity should be personalized and self-adaptive. Traditional methods of processing kinematic signals (obtained by multiple sensors) or bioelectrical signals (EMG, ECG) seem to be hard to couple the nonlinear relationship. To achieve rapid responses from rehabilitation robots, the control system needs to respond in real-time. Deep learning provides more possibilities for this response[20, 21]. 
Some scholars have proposed a transfer learning (TL) algorithm[22] that obtains rich data among users by mixing models of multiple topics. This mapping relationship can realize online data analysis for new objects. The Sensor-Wise method[23] achieves a high-precision and lightweight structure with only two hidden layers, but this method has limitations in the depth and accuracy of the motion intensity classification. On the whole, the use of physiological signals may be hampered by individual differences that are not easy to model, and there is a problem of hysteresis in kinematic signals[24]. From another perspective, the kinematic signals were used to sense the motion state and detect the deviation of the motion trajectory to achieve trajectory tracking[25]. Therefore, the deep learning algorithm is used to construct the multi-mode vector composed of physiological signals and kinematic signals to build the nonlinear coupling relationship among the multi-mode information[26, 27].

\section{Methodology}

\subsection{Datasets}

The ECG signals of 24 able-bodied subjects were collected at a sampling rate of $512 \mathrm{~Hz}$ using the BMD101 Bluetooth ECG acquisition module. At the same time, the angular velocity and linear acceleration of the joints are measured by an integrated industrial MEMS attitude sensor installed at the joints of elbow and shoulder of rehabilitation exoskeleton robotic arm. The MIVIE(Motion Intensity Variation Interval Estimation) dataset we set up was divided into two subdatasets, in which the data of 18 able-bodied subjects were used as training datasets to build models and optimize parameters, another 6 able-bodied subjects' data were used as test datasets for algorithm validation and trajectory optimization.

\subsubsection{ECG Recording Hardware.}

BMD101 is the 3rd generation bio-signal system-on-chip (SoC) of NeuroSky. Because of the BMD101's extremely low system noise and programmable gain, it can detect bio-signals and convert them into digital words using a 16-bit high resolution ADC.

As shown in Figure.1(a), BMD101 Bluetooth ECG sensor's sampling frequency is set at $512 \mathrm{~Hz}$ with the heart rate monitoring range of 24 200bpm that measurement error can be controlled within $\pm 1 \mathrm{bpm}$. In order to better observe the quality of signal collection, a specific online ECG waveform display interface was designed shown in Figure.1(b). To avoid the noise and baseline deviation caused by wire connection during the intense motion, the subjects will perform the muscle contraction and joint flexion and extension using a single-lead remote Bluetooth transmission protocol which can effectively solve the problems.

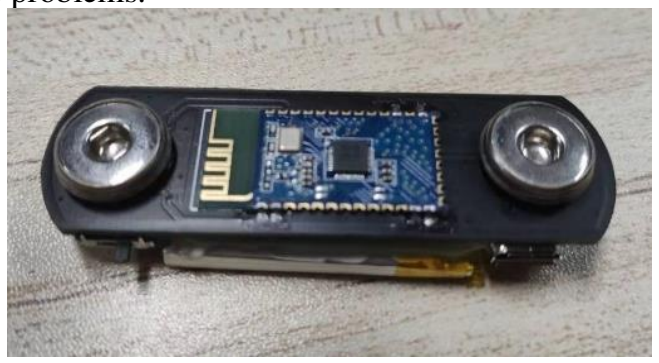

(a) BMD101 Bluetooth ECG sensor

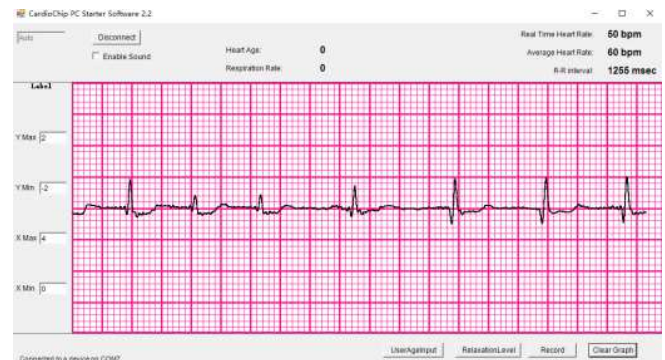

(b) Online ECG waveform display interface

Figure.1 BMD101 Bluetooth ECG acquisition module

\subsubsection{Time-window Length.}

In the processing of bioelectrical signal sequences, the performance of the classifier should take priority over the speed[28]. Recent studies[29] have shown that the Qi (Quality index) can measure classification performance. Furthermore, when there are fewer eigenvalues, a high Qi value can be obtained by using the Kaiser window function and the window length of $512 \mathrm{~ms}[29]$. In order to improve the classification efficiency[30], we choose the Kaiser window function with a maximum delay window of $256 \mathrm{~ms}$ to reduce the delay without affecting the classification performance. The 256 samples (256ms time window) separated by sliding window with the $32 \mathrm{~ms}$ sliding window step length and the $224 \mathrm{~ms}$ overlap allow approximately $15 \mathrm{~s} \sim 20 \mathrm{~s}$ for window data pre-processing.

\subsubsection{Data Acquisition Methods.}

Subjects must be trained before they formally participate in the data collection project. The collected data includes ECG at $512 \mathrm{~Hz}$ sampling frequency and a joint line acceleration and angular velocity signal at $60 \mathrm{~Hz}$ sampling frequency. The dataset named MIVIE is divided into three groups: SI (Strong Intensity), MI (Moderate Intensity), WI (Weak Intensity).

Specific Standards and rules for each group are shown in Table 1. Group SI exercise protocol included 30 seconds of warming up, 120 seconds of running at $9 \sim 12 \mathrm{~km} / \mathrm{h}, 30$ seconds of rest, 120 seconds of joint flexion/extension at 2 times/s, and 30 seconds of walking to relax. Group MI exercise protocol included 30 seconds of warming up, 100 seconds of running at $6 \sim 8 \mathrm{~km} / \mathrm{h}, 30$ seconds of rest, 90 seconds of arm lifting at 1 times/s, and 50 seconds of walking to relax. Group WI exercise protocol included 30 seconds of warming up, 80 seconds of running at $3 \sim 4 \mathrm{~km} / \mathrm{h}, 45$ seconds of rest, 40 seconds of arm lifting at 0.6 times/s, and 50 seconds of walking to relax.

In order to prevent muscle fatigue caused by excessive exercise during one day, each subject completed 15 sets of motion intensity training at intervals in a 3 -week period in which 24 participants were recorded.

Table 1. Standard and Rules of Different Training Motion Intensity Group

\begin{tabular}{|c|c|c|c|c|c|c|}
\hline $\begin{array}{c}\text { Gr } \\
\text { oup }\end{array}$ & $\begin{array}{l}\text { War } \\
\text { ming } \\
\text { up(s) }\end{array}$ & $\begin{array}{c}\text { Running } \\
\text { (s) }\end{array}$ & $\begin{array}{c}\mathrm{Re} \\
\mathrm{st} \\
(\mathrm{s})\end{array}$ & $\begin{array}{c}\text { Joint } \\
\text { flexion/e } \\
\text { xtension } \\
\text { (s) }\end{array}$ & $\begin{array}{c}\mathrm{W} \\
\text { alk } \\
\text { ing } \\
(\mathrm{s})\end{array}$ & $\begin{array}{l}\text { Effective } \\
\text { indicators }\end{array}$ \\
\hline SI & 30 & $\begin{array}{c}120(9 \sim 12 \\
\mathrm{km} / \mathrm{h})\end{array}$ & 30 & $\begin{array}{c}120(2 \\
\text { times/s })\end{array}$ & 30 & $\begin{array}{c}85 \%+\mathrm{HR} \max / 80 \\
\% \mathrm{VO} 2 \max \end{array}$ \\
\hline MI & 30 & $\begin{array}{c}100(6 \sim 8 \mathrm{k} \\
\mathrm{m} / \mathrm{h})\end{array}$ & 30 & $\begin{array}{c}90(1 \\
\text { times/s })\end{array}$ & 50 & $\begin{array}{l}60 \% \sim 70 \% \mathrm{HRma} \\
\mathrm{x} / 65 \%+\mathrm{VO} 2 \mathrm{max}\end{array}$ \\
\hline WI & 30 & $\begin{array}{c}80(3 \sim 4 \mathrm{k} \\
\mathrm{m} / \mathrm{h})\end{array}$ & 45 & $\begin{array}{l}40(0.6 \mathrm{tim} \\
\mathrm{es} / \mathrm{s})\end{array}$ & 50 & $\begin{array}{l}45 \% \sim 60 \% \mathrm{HRma} \\
\mathrm{x} / 50 \%+\mathrm{VO} 2 \mathrm{max}\end{array}$ \\
\hline
\end{tabular}


Effective indicators must meet any of the following requirements. Otherwise, it will be classified as invalid data:

(1).The average heart rate during exercise reaches the maximum heart rate of $85 \% / 60-75 \% / 45 \%-60 \%$.

(2). $\mathrm{VO}_{2} \max ($ Maximum oxygen uptake) reaches $80 \% / 65 \% / 50 \%$.

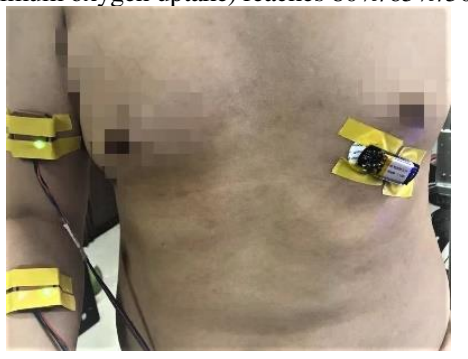

Figure.2 Rehabilitation scenario

The sensor placement on the body is shown in Figure 2. For the classification of the weak motion intensity, the test subject hardly takes the initiative to exercise his or her limbs during the entire rehabilitation exercise process and can complete the entire exercise track. For the classification of the moderate motion intensity, the maximum output torque of the actuator is limited, and the exercise can just barely be undertaken under no-load conditions. For the classification of the strong motion intensity, the output torque of the actuator on the rehabilitation robot arm is further restricted, and it can hardly drive itself to perform the rehabilitation exercises. In this case, the subject needs to actively exert force to complete the entire rehabilitation exercise trajectory.

All methods were carried out in accordance with relevant guidelines and regulations. All subjects' informed consent were confirmed by themselves. The data acquisition protocol was approved by Medical and Experimental Animal Ethics Committee of Northwestern Polytechnical University, Xi'an, China (approbation number: 6101030222595-202001001).

\subsection{Signal Pre-processing}

\subsubsection{Time Domain and Frequency Domain Analysis of ECG and Kinematics Signal}

Like other physiological signals, ECG is a kind of lowfrequency weak signal under strong noise background and has the following characteristics: The general sampling value of normal ECG fluctuates from $0.05 \mathrm{mv}$ to $5 \mathrm{mv}$, the frequency range is from $0.05 \mathrm{~Hz}$ to $100 \mathrm{~Hz}$, and $90 \%$ of ECG spectrum energy is concentrated from $0.25 \mathrm{~Hz}$ to $35 \mathrm{~Hz}$ [31].

The process of collecting ECG signal will be disturbed by various kinds of noise, the noise sources are usually as follows:

(1) Power-line interference: $50 \mathrm{~Hz}$ Power-line interference is caused by the electromagnetic field formed by the lead device which collects HR and the loop circuit of human body.

(2) EMG interference: The EMG interference can be regarded as the instantaneous zero mean band limited noise, and the main energy is concentrated in the range of 30 $300 \mathrm{~Hz}$.

(3) Baseline wander: Baseline wander in ECG amplitudes is generally caused by low frequency interference[32], such as aspiration and electrode movement, and the frequency is less than $5 \mathrm{HZ}$; the change can be regarded as a sine component added to HR at the same frequency as the aspiration frequency, at $0.015 \sim 0.03 \mathrm{~Hz}$, the amplitude of baseline change was $15 \%$ of the ECG peak value.

As shown in Table 2, the information of motion state mainly concentrates on P-T wave and QRS complex wave of ECG[33]. The frequency band of QRS complex wave band is
$3 \sim 40 \mathrm{~Hz}$, and the frequency band of P-T wave is $3 \sim 10 \mathrm{~Hz}$, so the interval frequency is filtered to reduce the noise.

Table 2. Frequency band distribution of main information and noise of ECG

\begin{tabular}{ccccc}
\hline \hline $\begin{array}{c}\text { Baseline } \\
\text { wander }\end{array}$ & $\begin{array}{c}\text { P-T } \\
\text { wave }\end{array}$ & $\begin{array}{c}\text { QRS } \\
\text { complex } \\
\text { wave }\end{array}$ & $\begin{array}{c}\text { Power-line } \\
\text { interference }\end{array}$ & $\begin{array}{c}\text { EMG } \\
\text { interference }\end{array}$ \\
\hline $0 \sim 0.5$ & $3 \sim 10$ & $3 \sim 40$ & 50 & $30 \sim 300$
\end{tabular}

For the nonlinear and non-stationary weak signal with strong randomness and noise, the traditional method has poor effect. Considering the characteristics of HR signal and the speed of operation, this paper chooses wavelet threshold denoising method and sets threshold function:

$$
\left\{\begin{array}{l}
W^{*}= \begin{cases}W-\alpha \lambda \frac{1-3^{W}}{1+3^{W}} & |W| \geq \lambda \\
0.01 & |W| \leq \lambda\end{cases} \\
\lambda=\frac{\sigma \sqrt{2 \ln N}}{\ln (j+1)} \\
\sigma=\frac{\operatorname{med}(|d|)}{E}
\end{array}\right.
$$

where $W$ is primitive wavelet coefficient, $W^{*}$ is the adaptive modified wavelet coefficient, $\alpha$ is the correction factor which is set to $0.4, \lambda$ is the threshold setting value, $N$ is the amount of ECG sampling points, $\sigma$ is the noise intensity estimation under the wavelet coefficient, $\operatorname{med}(|d|)$ is the median of the absolute value of the wavelet coefficients at each scale, $\mathrm{E}$ is the correction constant 0.65 .

ECG signals with noise was decomposed into 8 levels of wavelet by Coif 4 wavelets, then baseline wander was removed by wavelet decomposition and reconstruction. EMG and power frequency interference were removed by improved threshold algorithm, the specific effect of noise removal is shown in Figure.3.

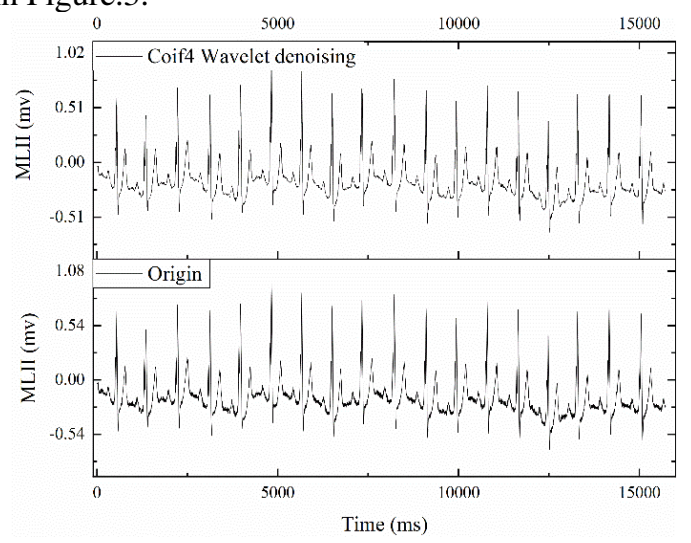

Figure.3 Comparison of denoising effect of improved Coif 4 wavelet threshold algorithm and original signal

\subsubsection{Extraction of ECG Eigenvalues}

The following eigenvalues were extracted as the input layer of the subsequent deep learning model.

- $\quad$ The standard deviation of the time domain $(S D)$ :

$$
S D=\sqrt{\frac{\sum_{i=1}^{N}\left|x_{i}-\mu\right|^{2}}{N}}
$$


where $x_{i}$ is the signal value corresponding to the time series, $N$ is the total number of sampling points of the time series, and $\mu$ is the average value of the segment signal.

\section{- Approximate Entropy (ApEn):}

The approximate entropy is a nonlinear dynamic parameter used to quantify the regularity and unpredictability of time series fluctuations.

For a one-dimensional series such as a heart rate, the extraction method was designed to reconstruct the vector and calculate the vector distance. Then, we counted the number of conformances and expressed series in the form of non-negative numbers. The algorithm hyper parameter $r=0.2 * S D$, which represents the parameter measure of "similarity", was used in this paper to reconstruct the vector dimension. The specific algorithm for the approximate entropy is expressed as follows.

The one-dimensional heart rate discrete signal obtained by sampling at equal intervals is $a(1), a(2) \ldots, a(N)$. we reconstituted the signal into a 3-dimensional vector, namely, $A(1), A(2) \ldots, A(N-m+1)$. When $1 \leq \mathrm{i} \leq \mathrm{N}-\mathrm{m}+1$, the number of reconstruction vectors satisfying the following conditions is:

$$
C_{i}^{m}(r)=\left[\begin{array}{l}
\text { number of } A(j) \text { such that } \\
d[A(i), A(j)] \leq r
\end{array}\right] /(N-m+1)
$$

where $d[A(i), A(j)]$ is the vector distance. The vector distance is defined as the value of the largest absolute difference of each dimension in the two reconstructed vectors, where the range of $j$ is $[1, N-m+1]$, including $j=i$.

$$
\phi_{r}^{m}(r)=(N-m+1)^{-1} \sum_{i=1}^{N-m+1} \log \left(C_{i}^{m}(r)\right)
$$

The state quantity of the current reconstructed dimension was defined by the above equation. The approximate entropy $(A p E n)$ equals the difference value between the state quantity of the strong reconstruction dimension and the current dimension state quantity.

$$
A p E n=\phi^{m}(r)-\phi^{m+1}(r)
$$

\section{- Frequency domain eigenvalues (FDE).}

Considering the real-time nature of the control accuracy, the frequency domain signal was obtained by a fast Fourier transform (FFT). We mainly used the root mean square frequency (RMSF) and the root variance frequency (RVF) as the frequency domain eigenvalues. These two parameters reflect the characteristics of the motion-related aspects more effectively. The specific algorithm is as follows.

$$
\begin{gathered}
R M S F=\sqrt{\frac{\int_{0}^{+\infty} f^{2} S(f) d f}{\int_{0}^{+\infty} S(f) d f}} \\
F C=\frac{\int_{0}^{+\infty} f S(f) d f}{\int_{0}^{+\infty} S(f) d f} \\
R V F=\sqrt{\frac{\int_{0}^{+\infty}(f-F C)^{2} S(f) d f}{\int_{0}^{+\infty} S(f) d f}}
\end{gathered}
$$

where $S$ is the corresponding frequency domain amplitude, $f$ is the corresponding frequency, $R M S F$ is the Root Mean Square Frequency, $F C$ is the frequency centre, and $R V F$ is the Root Variance Frequency.

\section{- Extraction of kinematic eigenvalues:}

The angle signal and the angular velocity signals of this article were obtained by the encoder of the INNFOS disc motor. Due to the diversification of the motion of the rehabilitation exercise, the joint angular velocity was also changed under different motions. Therefore, it was difficult to determine the specific motion intensity by directly determining the angular acceleration signal. Hence, we calculated the motion velocity of the collected data set based on the collected signal points in this paper.

$$
\begin{gathered}
\left\{\begin{array}{l}
R_{m}(i)=[r(i-m), r(i-(m-1)), \ldots, r(i)] \\
Y_{m}(i)=[y(i-m), y(i-(m-1)), \ldots, y(i)]
\end{array}\right. \\
A V D=\frac{\sum_{i=0}^{N} \mathrm{R}_{\mathrm{m}}(i)-\mathrm{Y}_{m}(i)}{N}
\end{gathered}
$$

where $R_{m}(i)$ is the one-dimensional vector composed of the measured angular velocity values in the previous $\mathrm{m}$ seconds, $Y_{m}(i)$ is the one-dimensional vector of the angular velocity value of the expected motion trajectory in the time period and $\mathrm{N}$ is the total number of the vectors.

The angular velocity deviation $(A V D)$ was obtained by the collected data set and the actual feedback signal. In summary, the process block diagram of building a multi-mode vector is described in Figure.4.

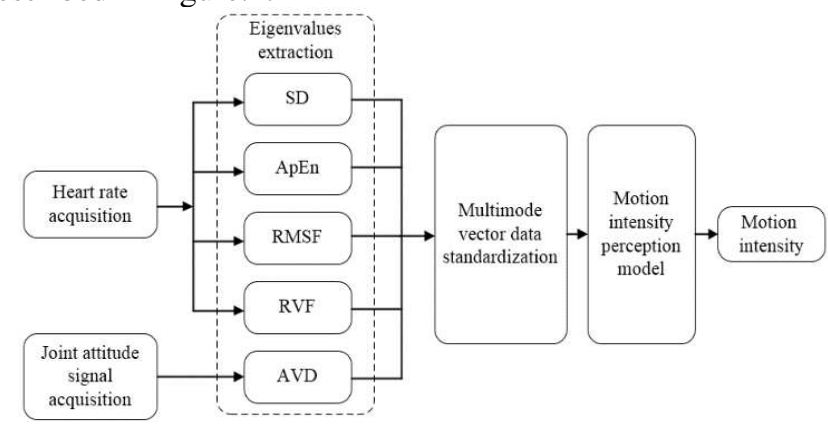

Figure.4 Multimode vector diagram

\subsubsection{Transformation from Eigenvalue Data Matrix to Grayscale Pixel Matrix}

If the original one-dimensional time series data matrix is directly used as network input, the network input dimension is too large resulting in expensive network computation. After extracting the eigenvalues of the original signal, the original Matrix of the input data is normalized and aligned.

In this paper, a method is proposed to convert batch onedimensional time series to two-dimensional gray-scale images, that is, the original time-domain signal is the format of the one-dimensional data matrix, which is transformed into an $\mathrm{m} \times \mathrm{n}$ grayscale pixel matrix.

In order to keep the pixel matrix stable and reduce the computation time of the procedure of the transformation, the random principle of $\mathrm{N}$ choosing 3 is adopted before the stack of the eigenvalues. On the other hand, too much preprocessing will lose the small information of the eigenvalues such as the wave crests of the time-frequency series in the QRS complex, so only the grayscale, the open operation and the bicubic interpolation operations are used after the pixel matrix is generated.

Firstly, we need to extract a R-R sequence from a continuous signal as a sample, and the grayscale pixel matrix is transformed by the data matrix:

$$
C(i, j)=\frac{A(i, j)-\min (A)}{\max (A)-\min (A)} \times 255
$$


where $i$ is the ordinal number of eigenvalues, $j$ is the ordinal number of the sampling points of R-R time series, $\max / \min (\mathrm{A})$ represents the maximum and minimum values of the pixel matrix, and $\mathrm{C}$ matrix is the normalized matrix.

Figure. 5 shows the conversion process from eigenvalue data matrix to gray pixel matrix. After the normalization of the Matrix from 0 to 255 , the Pixel Matrix with dimension $n \times m$ is obtained. Secondly, the "square" structure element performs an open operation on the grayscale image to remove the process noise caused by the low signal-to-noise ratio. Finally, the time-frequency image with the size of $800 \times 800$ is adjusted to $227 \times 227$ by using the bicubic interpolation algorithm.

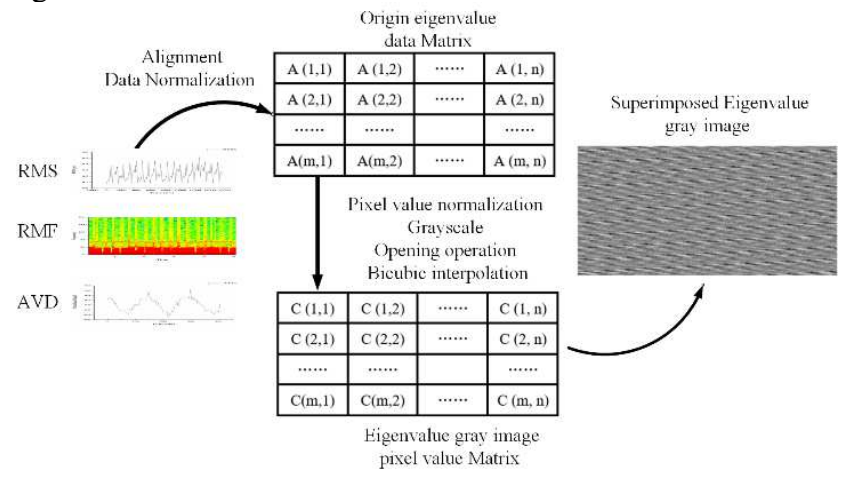

Figure.5 The conversion process from eigenvalue data matrix to gray pixel matrix

\subsection{Resampling of the Rehabilitation Motion Trajectory}

The rehabilitation motion trajectory collected from volunteers through the teaching reply mode could not be directly used to simulate the patient's experiment. The sampled trajectory points needed to be resampled to facilitate the adjustment of the motion rate during the experiment[34].

The Lanczos resampling method is widely used in the twodimensional vector resampling process in the field of image processing[35]. Considering the function mentioned above, the effect of smooth interpolation was available for the onedimensional data such as the rehabilitation motion trajectory data. The purpose of the resampling is to double the number of points in the rehabilitation motion

Let the input point be $x$. Then, the weight of the Lanczos window function corresponding to each point is defined as

$$
\begin{gathered}
\sin c(x)=\frac{\sin (\pi x)}{\pi x} \\
L(x)=\left\{\begin{array}{cc}
\sin c(x) \sin c(x / a) & \text { if }-a<x<a \\
0 & \text { otherwise }
\end{array}\right.
\end{gathered}
$$

where $a$ can be taken as 2 or 3 , as it is the hyper parameter that corresponds to the adjustment and reduction interpolation or the amplification interpolation. Because the purpose of this paper is to up sample, $a$ is set to 3 .

Then, the set of reconstructed points corresponding to the specific reconstruction function is defined as follows:

$$
S(x)=\sum_{i=|x|-a+1}^{|x|+a} s_{i} L(x-i)
$$

where $S(x)$ is the resampled value at the position, which is the sampled value of the original position.

\subsection{Motion Intensity Mutation Perception Model}

The input layer of the motion-intensity perception model consists of multi-modal information fusion data, and the expected output is the specific exercise intensity classification result. The deep neural network (DNN) method is used to implement the model.

\subsubsection{Neural Network Environment}

The programming uses the TensorFlow framework and the Keras library, trains the model in the Python environment, uses Nvidia's CUDA parallel computing architecture to achieve hardware acceleration, and uses the $\mathrm{C}++$ language API interface in the control software to connect the control program of the actuator.

\subsubsection{Architecture and Parameters of Inception-Sim model}

After using the ZCA whitening method to reduce the redundant information, the model takes the feature value stack map as input, and then sends it to the convolutional layer of $5 \times 5,3 \times 3$ convolution kernel, which is used to extract the fluctuation trend information of eigenvalues in the low frequency band.

The DropBlock layer can be used to simulate noise and improve generalization ability[36]. The traditional dropout regularization technique is not used in the proposed network, because for the convolution layer, the feature map adjacent position elements share semantics in the spatial block area, and the structured DropBlock layer performs better in the convolution network where mentioned in the Discussions and Results section.

$$
\gamma=\frac{1-k p}{b s^{2}} \frac{f s^{2}}{(f s-b s+1)^{2}}
$$

where $k p$ represents the probability of a unit remaining in the traditional dropout (between 0.75 and 0.95 , with a final value of 0.9$), f s$ represents the size of the feature map, $b s$ represents block size.

DropBlock has two main parameters, $b s$ is set to 7 , the $\gamma$ is $3.373 \times 10^{-3}$ caculated from equation 15 .

After the first two convolution layers, using the advantage of the Inception structure to reduce the number of parameters, Inception-sim is proposed for the small amount of classification (in our work, we need 6 classifications) based on the InceptionV3 convolution network structure.

Compared with traditional Inception-V3[37] module "one $5 \times 5$ convolution replaced by two $3 \times 3$ convolution" (" 5 th layer" module), Inception-Simin also added "N×1 and $1 \times N$ " module ("6th layer" module) to reduce the network overfitting and speed up the network, the specific acceleration fusion module is shown in Figure.6 


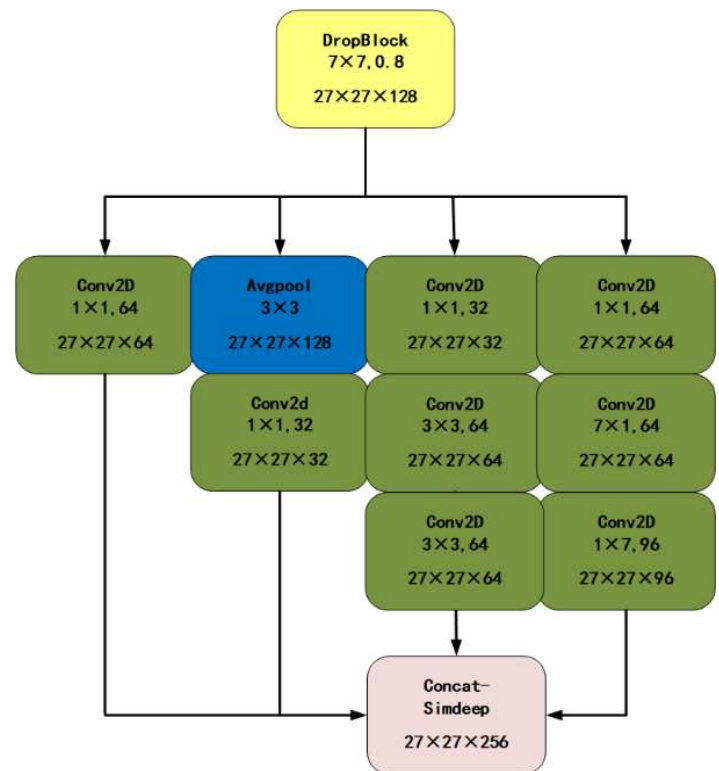

Figure.6 Inception-Simin module: From left to right, the third sub-column represents mini module of the " 5 th layer" module, the fourth sub-column represents mini module of "6th layer".

Inception-Simdeep shown in Figure.7 is used in deep layers and reduces the feature map size to increase network depth by combining of "6th layer" and "7th layer". An example is given to illustrate the meaning of "split or connect" indicated by the arrow. The $19 \times 19 \times 64$ layer of conv2d is connected to the $27 \times 27 \times 96$ Maxpool layer, and finally the $19 \times 19 \times 160$ layer of Maxpool layer is generated. Its advantage is that it realizes the function of inception to reduce the parameters, and can save the calculation time and improve the accuracy in part, which is discussed in the Result and Discussion section.

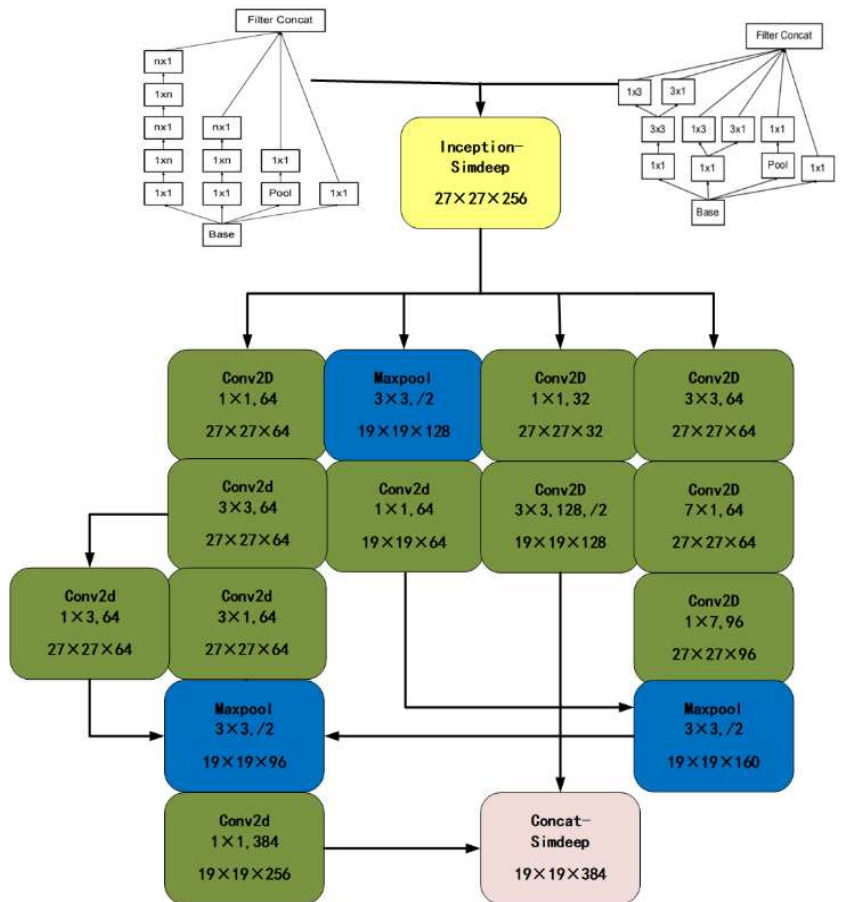

Figure.7 Inception-Simdeep module: "3×3" represents the size of kernel,"/2" represents stride of $2, " 64$ " represents the amount of kernel

Through the collaboration of Inception-Sim layer and DropBlock layer to achieve acceleration, the implementation of "first Inception-Sim and then "Avgpooling" mode is conducive to maintaining feature stability, but it increases the complexity of calculation by three times. Finally, through the whole connection layer Softmax output six kinds of motion intensity mutation mode, the specific network model is shown in the Figure. 8 and Table 3.

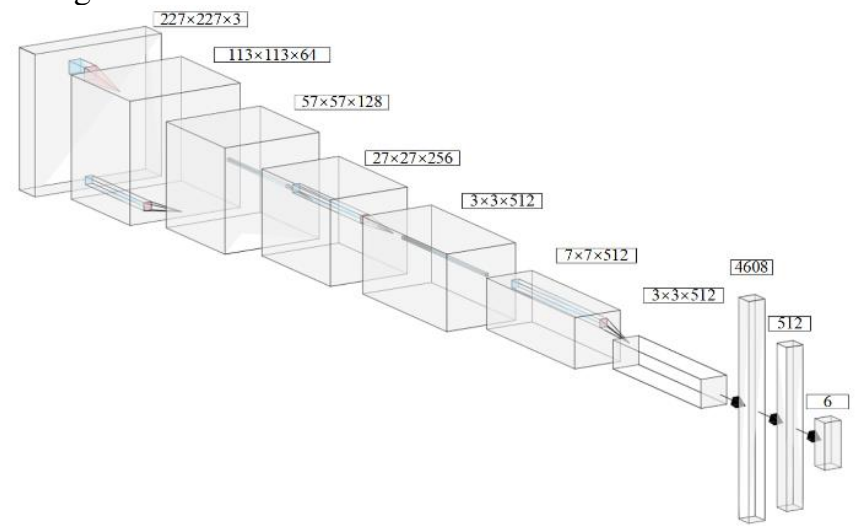

Figure.8 Motion intensity mutation perception model's network structure

TABLE 3. Summary of Motion Intensity Mutation Perception Model

\begin{tabular}{cccc}
\hline \hline Layer & $\begin{array}{c}\text { Output layer } \\
\text { dimension }\end{array}$ & $\begin{array}{c}\text { Kernel } \\
\text { dimension }\end{array}$ & Parameter \\
\hline Feature-in & $227 \times 227 \times 3$ & & \\
\hline Conv 1 & $113 \times 113 \times 64$ & $5 \times 5$ & Leaky_relu \\
Maxpool 1 & $57 \times 57 \times 64$ & $2 \times 2$ & Stride=2 \\
Conv 2 & $57 \times 57 \times 128$ & $3 \times 3$ & Leaky_relu \\
Maxpool 2 & $27 \times 27 \times 128$ & $2 \times 2$ & Stride=2 \\
DropBlock & $27 \times 27 \times 128$ & 7 & $\gamma=3.373 \mathrm{e}-3$ \\
\hline Inception-Simin & $27 \times 27 \times 256$ & & Relu \\
Inception- & $19 \times 19 \times 384$ & $1 \times 1 / 3 \times 3$ & Relu \\
Simdeep & $7 \times 7 \times 512$ & & Relu \\
Inception- & $7 \times 1 / 1 \times 7$ & Stride=2 \\
Simdeep & $3 \times 3 \times 512$ & $2 \times 2$ & $\gamma=3.373 \mathrm{e}-3$ \\
\hline AvgPool & $3 \times 3 \times 512$ & 1 & Dropout=0.9 \\
DropBlock & $1 \times 1 \times 4608$ & & Dropout=0.7 \\
\hline FC 1 & $1 \times 1 \times 512$ & & \\
FC 2 & $1 \times 1 \times 6$ & & \\
Softmax & &
\end{tabular}

\subsection{Adaptive trajectory optimization algorithm based on DQN}

Impedance control allows only the actual trajectory of the robot to be adjusted, rather than associated with the desired trajectory of the robot[38], which leads to the out of step of motor in the face of motion intensity mutation. The reason is that when the output of the joint motor does not reach the desired position, a new position control signal is received, and the original position control signal is overwritten, resulting in the deformation of the motion trajectory.

Faced with such problems, this paper proposes a trajectory optimization algorithm aiming at stabilizing motion intensity. The strategy is to optimize the dynamic trajectory in each time window, and adjust the joint drive motor speed based on DQN (Deep Q Network) algorithm to keep the motion intensity in the "moderate" state. With the iteration of the algorithm, the deviation between the actual trajectory and the desired trajectory gradually tends to 0 .

Markov decision process is generally described using Multituple ( $S, A, P, R, \gamma), S$ is finite state set, $A$ is action set, $P$ is finite state transition conditional probability, $r$ is reward function, $\gamma$ is discount factor of reward function, used to measure long-term reward and penalty.

- State Space:

$$
S_{i}=\left(U_{s}(i), U_{e}(i), D_{t}(i)\right)^{T}
$$


where $U_{s}(i)$ is the angle signal value converted by shoulder encoder, $U_{e}(i)$ is the angle signal value converted by the elbow encoder, $D_{e}(i)$ is the distance value between the target point and the current position in the Cartesian space coordinate system.

- Action Space:

$$
A_{t}=\left(\omega_{s}(t), \omega_{e}(t)\right)
$$

where $\omega_{s}(t), \omega_{e}(t)$ are the angular velocity of shoulder joint and elbow joint servo motor respectively, through the control of motor angular velocity to achieve state transfer.

- Reward Function:

$$
r_{s s^{\prime}}^{a}(i)=\left\{\begin{array}{cc}
\frac{-5}{D(i)\left[1+\left|\Delta U_{s}^{*}(i)+\Delta U_{e}^{*}(i)\right|\right]} & m=1,2 \\
\frac{50}{D(i)\left[1+\left|\Delta U_{s}^{*}(i)+\Delta U_{e}^{*}(i)\right|\right]} & m=3,4 \\
\frac{-10}{D(i)\left[1+\left|\Delta U_{s}^{*}(i)+\Delta U_{e}^{*}(i)\right|\right]} & m=5,6 \\
-5 \quad \sum_{j=i-3}^{i} r_{s s^{\prime}}^{a}(j)<0.75, D(i)>D(i-3) &
\end{array}\right.
$$

where $m$ represents the mutation type of motion intensity prediction (Table 4 shows the corresponding meaning of $m$ ) , $\Delta U_{s}(i), \Delta U_{e}^{*}(i)$ are differentials between the current position and the desired position of shoulder joint, elbow joint, respectively.

Table 4. Corresponding Meaning of the Value of $m$

\begin{tabular}{cccc}
\hline \hline Value of $m$ & 1,2 & 3,4 & 5,6 \\
\hline $\begin{array}{c}\text { Motion intensity } \\
\text { mutation types }\end{array}$ & $\mathrm{M} \rightarrow \mathrm{S}$ & $\mathrm{W} \rightarrow \mathrm{M}$ & $\mathrm{W} \rightarrow \mathrm{S}$ \\
\hline \hline
\end{tabular}

M(Moderate), W(Weak), S(Strong) represents the type of motion intensity mutation. The reward function rewards the maintenance of "moderate" intensity actions, and punishes motion intensity mutation actions. Moreover, the penalty of intensity mutation across two grades $(m=5,6)$ is much greater than that of across one grade $(\mathrm{m}=1,2)$ and its concrete effect is shown in the reward function.

The $\varepsilon$-greedy algorithm is used to select the action of the actual $Q$ value:

$$
\pi(a \mid s) \leftarrow \begin{cases}1-\varepsilon+\frac{\varepsilon}{k} & \text { if } \mathrm{a}=\underset{a}{\arg \max } q_{\pi}(s, a) \\ \frac{\varepsilon}{k} & \text { if } \mathrm{a} \neq \underset{a}{\arg \max } q_{\pi}(s, a)\end{cases}
$$

where $\varepsilon$ is the hyper-parameter of the algorithm; $\mathrm{k}($ set $\mathrm{k}=4)$ represents the amount of action types that the algorithm can choose.

In the specific implementation algorithm of trajectory optimization strategy based on DQN, the pseudo code is shown in Table 5.

Table 5. Algorithm: Adaptive Trajectory Optimization Algorithm based on $D Q N$

$1:$ For episode $=1$, do

2: Read the initial value of exoskeleton manipulator joint position and

motion intensity state

3: For $i=1$, do

4: With $\varepsilon$ probability random logic

True: Randomly selected action: $A_{i}$

False: Select the action corresponding to the maximum $\mathrm{Q}$ value

5: Execute corresponding trajectory optimization action $A_{t}$ and

calculate the reward function $r(i)$

6: Record the next time slice of the manipulator system: $S_{\mathrm{i}+1}$
7: $\quad$ Store the $(\mathrm{Si}, a i, r, \mathrm{Si}+1)$ data in experience replay

8: Set $y_{i}= \begin{cases}r_{i} & D(i)<0.01 \\ r_{i}+\gamma \max _{a_{i+1}} q\left(S_{i+1}, a_{i+1}, \theta^{\prime}\right) & \text { Otherwise }\end{cases}$

9: Gradient descent method to update network layer parameters for evaluating Q network: $L=\left(y_{i}-q\left(S_{i}, a_{i}, \theta\right)\right)^{2}$

10: Copy the network layer parameters of the evaluated network to the target network per completion of a training branch

11: end for

12 :end for

\section{Experimental verification}

\subsection{Demonstration teaching mode}

To realize the demonstration teaching mode, it is necessary to collect the motion track position signal through motion capture. When collecting motion track position signals, the INNFOS joint actuator is set to the "current" mode. In this state, the current in the actuator is zero, there is no holding torque, the upper limbs of a healthy person can directly drive the INNFOS joint actuator, and the integrated encoder will record the current movement track position data.

The motion trajectory is collected for the rehabilitation. The rehabilitation motion requires the coordination of the four main joints of the upper limbs of the human body. The encoder data has been converted into joint motion angle data. The zero position of the joint angle corresponds to the initial state, the elbow joint has degrees of freedom in flexion/extension, and the shoulder joints M1, M2 and M3 correspond to abduction/adduction, flexion/extension, and internal/external rotation degrees of freedom, respectively. The motion trajectory is shown in Figure.9.

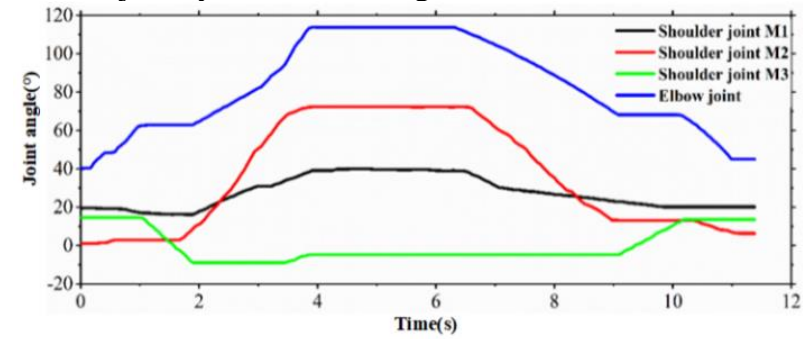

Figure.9 Motion trajectory of the shoulder and elbow joint angle

\subsection{Design of the Rehabilitation motion decomposition}

The motion intensity mutation perception model has achieved good results in offline testing, but the use of the model in actual scenarios requires the online model to achieve real-time interaction. The designed rehabilitation experiment is conducted on the two basic degrees of flexion/extension freedom of the shoulder and elbow joint. The specific action decomposition diagram we adopted is shown in Figure.10. The motion trajectory of the actuator is collected through the demonstration teaching mode as shown in Figure.11.

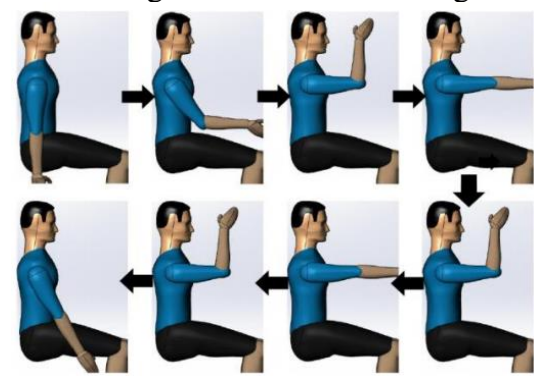

Figure.10 Rehabilitation motion decomposition 


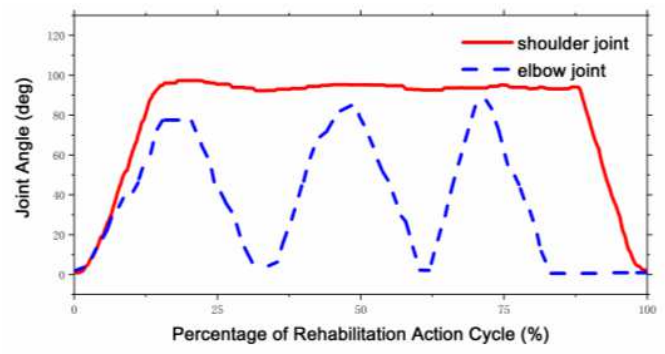

Figure.11 Rehabilitation motion trajectory through the demonstration teaching mode

As shown in Figure.12, the exoskeleton arm was fixed on the aluminium alloy steel frame. Additionally, the upper arm and the forearm were loaded with extra $1.0 \mathrm{~kg}$ weights. The simulated patient wore the exoskeleton arm by grasping the grip of this arm with the right hand. The nylon bolts were tightened to adjust the overall length to a comfortable location. The heart rate sensor was worn on the patient's left-hand finger. After confirmation that the setup was correct, we turned on the power for the rehabilitation experiment. This experiment was conducted in five consecutive rehabilitation cycles with a pause of 5 seconds after each cycle.

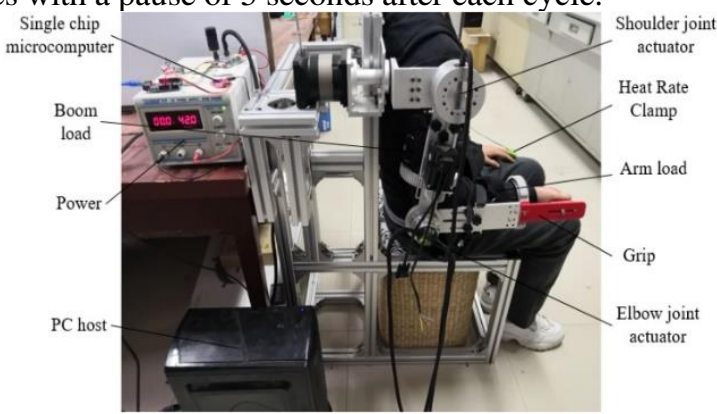

Figure.12 Rehabilitation motion experimental platform

\subsection{Verification and optimization of the motion- intensity perception model1.1 Subsection heading}

The transformation from an offline model to an online model requires optimization of the program packaging interface and running time. The engineering optimization is mainly to use the CPU multi-threading feature, which can encapsulate the perception model and translate it into the Visual Studio 2017 platform. The control program uses the reserved input layer and output layer program interfaces. After the test, a thread is established in the main program for data collection, and multi-mode fusion and standardization are performed. The running time of identification using the perception model is approximately $1.8 \mathrm{~s}$, the thread resource occupancy rate is in line with expectations, and the heart rate signal acquisition cycle is 3 seconds. In the designed experiment, the motion intensity will be initially set to the medium intensity, and the online perception model will correct the real-time exercise intensity every 5 seconds. The encoder records five consecutive cycles of motion trajectory, which are shown in Figure.13.

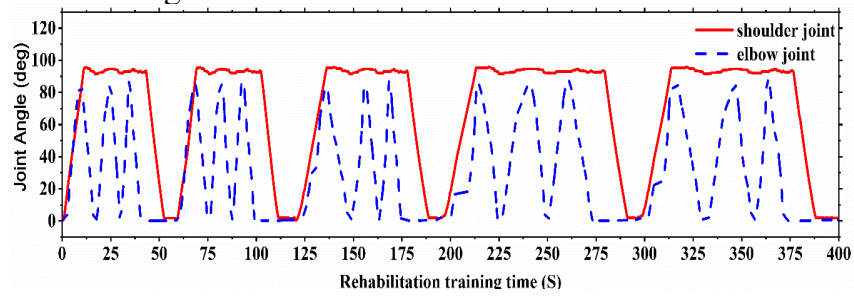

Figure.13 Experimental motion trajectory of five consecutive cycles
The data obtained by the online motion intensity mutation perception model are shown in Table 6 . The results show that in the first three rehabilitation cycles, the motion intensity of the subjects is mainly concentrated in the mutual transformation between "strong" and "moderate". The main reason is that the subjects are full of energy at the beginning of the experiment, producing a strong interactive force between the muscles and the manipulator. During the last two cycles, the subjects' motion intensity gradually changed from "moderate" to "weak", accompanied by a decrease in motion velocity and deformation of the motion trajectory, which was caused by the subject's muscle fatigue and a decrease of upper limb strength.

In summary, the data obtained by the online perception model are basically consistent with the motion trajectory measured in the experiment. The classification accuracy of the perception model has been verified, laying a foundation for the adaptive trajectory optimization algorithm based on DQN.

Table 6. Data of Online Motion Intensity Perception Model

\begin{tabular}{cccccc}
\hline Intensity & $\begin{array}{c}\text { The } \\
\text { first }\end{array}$ & $\begin{array}{c}\text { The } \\
\text { second }\end{array}$ & $\begin{array}{c}\text { The } \\
\text { third }\end{array}$ & $\begin{array}{c}\text { The } \\
\text { fourth }\end{array}$ & $\begin{array}{c}\text { The } \\
\text { fifth }\end{array}$ \\
\hline Strong & $25.2 \%$ & $24.3 \%$ & $6.6 \%$ & $0.0 \%$ & $0.0 \%$ \\
Moderate & $74.8 \%$ & $75.9 \%$ & $65.3 \%$ & $13.4 \%$ & $18.8 \%$ \\
Weak & $0.0 \%$ & $0.0 \%$ & $28.1 \%$ & $86.6 \%$ & $81.2 \%$ \\
\hline \hline
\end{tabular}

\section{Experimental verification}

\subsection{Multi-mode information fusion}

The multi-mode feature vector constructed in this paper was composed of ECG eigenvalues and kinematic eigenvalues. A parallel coordinate plot adopting the standardized preprocessing is shown in Figure.14(a). For the intensity label, the solid line indicates that the prediction vector of the model matches the actual label, while the dashed line is opposite. From the perspective of feature value dimension, the feature vectors of four-dimensional heart rate signal are densely distributed with high accuracy, while the feature vectors of two-dimensional kinematic signal are distributed dispersedly, which is roughly due to the relative hysteresis and anisotropy of the kinematic signal.

The vectors preprocessed by standardization are more densely distributed under the same label and more dispersed under different labels, which is convenient for classification and identification of the model. In contrast, the vectors shown in Figure.14(b) that are preprocessed by normalization are scattered under the same label, mainly because normalization does not change the relative order of the data, but it does not keep the space between the dimensions of the initial vector. Finally, the gradient identification of each dimension of the initial vector disappears.

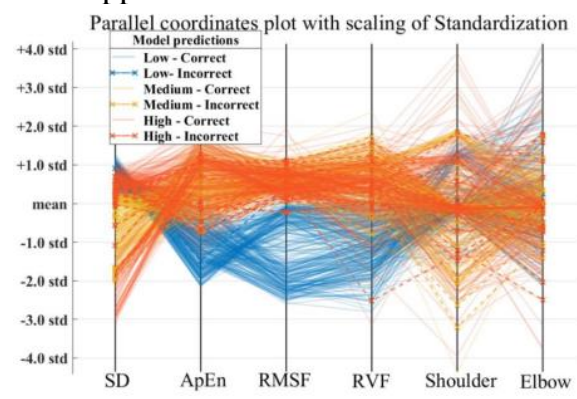

(a). Parallel coordinates plot with scaling of Standardization 


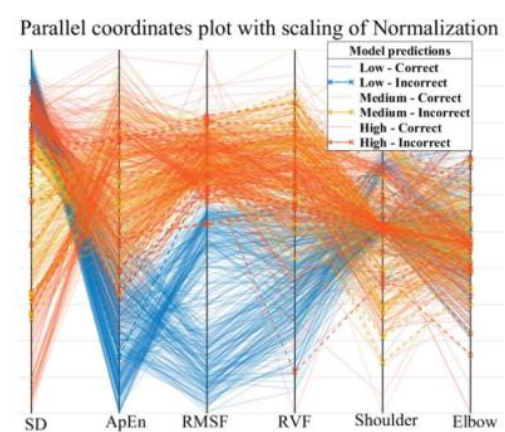

(b). Parallel coordinates plot with scaling of Normalization

Figure.14 Parallel coordinates plot with scaling of Standardization and Normalization

\subsection{Performance evaluation of proposed model}

\subsubsection{Baselines}

In order to verify the performance of the proposed model in individual motion intensity mutation monitoring, the multiple state-of-the-art methods are listed below for comparison:

MLP-CNN[39]: The model includes two algorithms which were integrated in a concise and effective way using a rulebased decision fusion approach.

ECG-CNN[40]: The deep two-dimensional CNN for ECG arrhythmia classification using the Xavier initialization and exponential linear units.

VGGNET[41]:The model uses very small $(3 \times 3)$ convolution filters thorough evaluation of networks with the depth to 16-19 weight layers.

34-layer CNN[42]: The network contains 33 layers of convolution followed by a fully connected layer and a softmax.

Besides the above networks, we also compare with widelyused image classification frameworks, such as VGG16, Resnet50 and Inception-V3.

\subsubsection{Evaluation index}

Precision, Recall, ACR (average convergence rate) and F1 are used as network performance evaluation indexes, which are expressed as follows:

$$
\begin{aligned}
& \text { Precision }=\frac{T P}{T P+F P} \\
& \text { average convergence rate }=\frac{1}{\text { epoch time }} \\
& \text { Recall }=\frac{T P}{T P+F N} \\
& F 1=2 \times \frac{\text { Precision } \times \text { Recall }}{\text { Precision }+ \text { Recall }}
\end{aligned}
$$

where epoch time represents the overall time when the loss function value stabilizes, TP FP TN FN represents for True Positives, False Positives, True Negatives and False Negatives, respectively.

\subsubsection{Comparison with the state-of-the-art methods}

We compared the proposed method with the latest method, and tested on MIT-BIH ECG Compression Test database (CDB) and MIVIE database(collected by experiments), respectively. To save space, we show two typical classification labels (W to $\mathrm{S}$, to $\mathrm{S}$ ) precision and recall (left of the table), overall (right of the table) precision, recall, F1 scores and ACR.

\begin{tabular}{|c|c|c|c|c|c|c|}
\hline $\begin{array}{c}\text { Intensit } \\
\mathrm{y}\end{array}$ & $\begin{array}{c}\text { W to } S \\
\text { Precisi } \\
\text { on/Rec } \\
\text { all }\end{array}$ & $\begin{array}{l}\text { M to } S \\
\text { Precisi } \\
\text { on } / \operatorname{Rec} \\
\text { all }\end{array}$ & $\begin{array}{c}\text { Overall } \\
\text { Precisi } \\
\text { on }\end{array}$ & $\begin{array}{c}\text { Overall } \\
\text { Recall }\end{array}$ & $\mathrm{F} 1$ & $\begin{array}{c}\text { ACR } \\
(1 / \mathrm{s} \\
)\end{array}$ \\
\hline $\begin{array}{l}\text { MLP- } \\
\text { CNN }\end{array}$ & $\begin{array}{c}0.6145 / \\
0.6568\end{array}$ & $\begin{array}{l}0.7985 / \\
0.6125\end{array}$ & 0.6342 & 0.5852 & 0.6087 & $\begin{array}{c}4.032 \mathrm{e}- \\
3\end{array}$ \\
\hline $\begin{array}{l}\text { ECG- } \\
\text { CNN }\end{array}$ & $\begin{array}{c}0.7652 / \\
0.4823\end{array}$ & $\begin{array}{c}\mathbf{0 . 9 4 5 6 /} \\
0.6935\end{array}$ & 0.8654 & 0.6561 & 0.7463 & $\begin{array}{c}2.932 \mathrm{e}- \\
3\end{array}$ \\
\hline $\begin{array}{c}\text { VGGN } \\
\text { ET }\end{array}$ & $\begin{array}{c}0.8211 / \\
\mathbf{0 . 7 5 2 0}\end{array}$ & $\begin{array}{c}0.6425 / \\
0.5354\end{array}$ & 0.7524 & 0.5628 & 0.6439 & $\begin{array}{c}3.932 \mathrm{e}- \\
3\end{array}$ \\
\hline $\begin{array}{l}34- \\
\text { layer } \\
\mathrm{CNN}\end{array}$ & $\begin{array}{l}0.8452 / \\
0.6245\end{array}$ & $\begin{array}{l}0.8012 / \\
0.6723\end{array}$ & 0.8124 & 0.6724 & 0.7358 & $\begin{array}{c}4.932 \mathrm{e}- \\
3\end{array}$ \\
\hline $\begin{array}{l}\text { Propos } \\
\text { ed } \\
\text { method }\end{array}$ & $\begin{array}{c}\mathbf{0 . 9 2 2 3} / \\
0.6123\end{array}$ & $\begin{array}{l}0.8145 / \\
0.2453\end{array}$ & 0.8721 & 0.6242 & 0.7276 & $\begin{array}{c}5.132 \mathrm{e}- \\
3\end{array}$ \\
\hline
\end{tabular}
In all experiments and evaluations of the proposed method is based on the results of 5-fold cross-validation.
Table 7. Model Evaluation On MIT-BIH ECG Compression Test Database(CDB)

\begin{tabular}{|c|c|c|c|c|c|c|}
\hline $\begin{array}{c}\text { Intensi } \\
\text { ty }\end{array}$ & $\begin{array}{c}\mathrm{W} \text { to } \mathrm{S} \\
\text { Precisi } \\
\text { on/Rec } \\
\text { all } \\
\end{array}$ & $\begin{array}{c}\text { M to } S \\
\text { Precisi } \\
\text { on/Rec } \\
\text { all } \\
\end{array}$ & $\begin{array}{c}\text { Overall } \\
\text { Precisi } \\
\text { on }\end{array}$ & $\begin{array}{c}\text { Overall } \\
\text { Recall }\end{array}$ & $\mathrm{F} 1$ & $\begin{array}{c}\text { ACR } \\
(1 / \mathrm{s} \\
)\end{array}$ \\
\hline $\begin{array}{l}\text { MLP- } \\
\text { CNN }\end{array}$ & $\begin{array}{c}0.8522 / \\
0.8268\end{array}$ & $\begin{array}{c}0.7565 / \\
0.7245\end{array}$ & 0.8245 & 0.7852 & 0.8043 & $\begin{array}{c}4.724 \mathrm{e}- \\
2\end{array}$ \\
\hline $\begin{array}{l}\text { ECG- } \\
\text { CNN }\end{array}$ & $\begin{array}{c}0.8678 / \\
0.8521\end{array}$ & $\begin{array}{c}0.8785 / \\
0.6732\end{array}$ & 0.8425 & 0.7541 & 0.7958 & $\begin{array}{c}5.584 \mathrm{e}- \\
2\end{array}$ \\
\hline $\begin{array}{c}\text { VGGN } \\
\text { ET }\end{array}$ & $\begin{array}{c}0.8842 / \\
0.8289\end{array}$ & $\begin{array}{c}\mathbf{0 . 9 7 5 2} / \\
0.8354\end{array}$ & 0.9124 & 0.5628 & 0.6961 & $\begin{array}{c}6.4263 \\
2 \mathrm{e}-2\end{array}$ \\
\hline $\begin{array}{l}34- \\
\text { layer } \\
\mathrm{CNN}\end{array}$ & $\begin{array}{c}0.8452 / \\
0.6245\end{array}$ & $\begin{array}{c}0.8012 / \\
0.6723\end{array}$ & 0.8124 & 0.6842 & 0.7428 & $\begin{array}{c}7.546 \mathrm{e}- \\
2\end{array}$ \\
\hline $\begin{array}{c}\text { VGG1 } \\
6\end{array}$ & $\begin{array}{c}0.8183 / \\
0.6423\end{array}$ & $\begin{array}{c}0.8523 / \\
0.6247\end{array}$ & 0.8345 & 0.6423 & 0.7258 & $\begin{array}{c}5.542 \mathrm{e}- \\
2\end{array}$ \\
\hline $\begin{array}{c}\text { Resnet } \\
50\end{array}$ & $\begin{array}{c}0.8745 / \\
0.8523\end{array}$ & $\begin{array}{c}0.9123 / \\
0.7825\end{array}$ & 0.8915 & 0.8354 & 0.8625 & $\begin{array}{c}7.678 \mathrm{e}- \\
2\end{array}$ \\
\hline $\begin{array}{l}\text { Incepti } \\
\text { on-V3 }\end{array}$ & $\begin{array}{c}0.8824 / \\
\mathbf{0 . 8 5 2 4}\end{array}$ & $\begin{array}{c}0.9254 / \\
0.7852\end{array}$ & 0.9041 & 0.8123 & 0.8557 & $\begin{array}{c}6.852 \mathrm{e}- \\
2\end{array}$ \\
\hline $\begin{array}{l}\text { Propos } \\
\text { ed } \\
\text { metho } \\
\text { d }\end{array}$ & $\begin{array}{c}\mathbf{0 . 8 9 5 2} / \\
0.8123\end{array}$ & $\begin{array}{c}0.9345 / \\
0.7845\end{array}$ & 0.9081 & 0.7563 & 0.8252 & $\begin{array}{c}9.132 \mathrm{e}- \\
2\end{array}$ \\
\hline
\end{tabular}

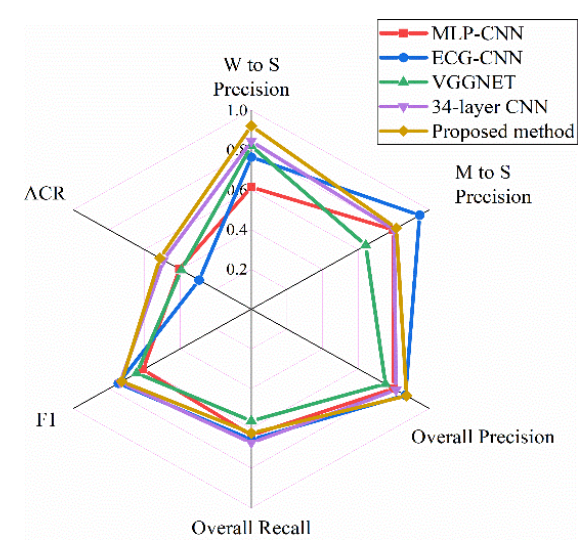

Figure.15 Radar chart of classification effect of CDB dataset Table 8. Model Evaluation On MIVIE Database 


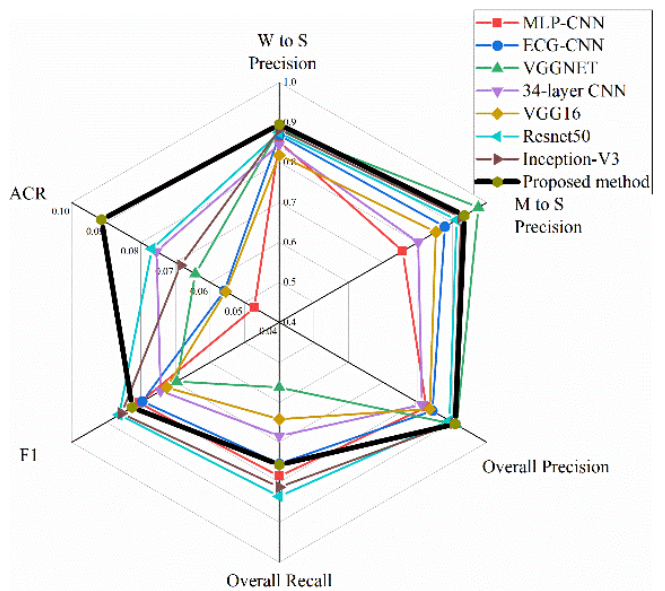

Figure.16 Radar Chart of Classification Effect of MIVIE Dataset

Results are demonstrated in Table 7, Table 8, respectively. In order to observe the results more intuitively, we visualize the model performance evaluation into Radar Chart shown in Figure 15 and Figure 16 through data visualization. On the whole, poor performance of classification methods based on time series (ECG-CNN and 34-layer CNN) can be observed in both datasets. It is worth noting that the general image classification framework (VGG16, inception-v3, Resnet50) achieves better performance than these digital-based approaches. This situation is in line with our original intention of transformation from eigenvalue data matrix to grayscale pixel matrix, that is, the application of time series data to image classification framework will be limited. When the proposed method comparing with these conventional image classification frameworks, index of Precision, F1, and Recall remains at a high level but the most prominent performance index is ACR, the structure optimization of our improved inception-CNN network plays a key role in reducing redundant network parameters and improving network running time.

\subsection{Analysis of individual differences}

It is not sufficiently comprehensive to analyse the problem of individual differences based on the classification accuracy of the perception model. We randomly extract 100 sets of data from each measured exercise intensity label, and we use the tdistributed stochastic neighbour embedding (t-SNE) method to perform dimensionality reduction and visualization of the data. The t-SNE method is used to reduce the dimensionality of the multi-mode fusion vector to two dimensions. The twodimensional distribution graphics are shown in Figure.17.
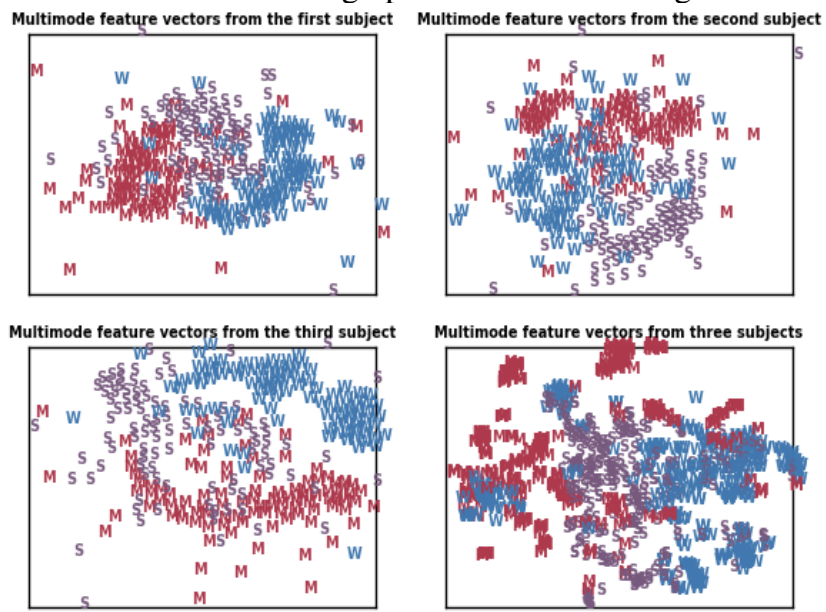

Figure.17 t-SNE embeddings of multimode feature vectors from different subjects

The different colours of W(Weak), M(Moderate), and $\mathrm{S}$ (Strong) in the figure represent the multi-mode vector of weak motion intensity, moderate motion intensity and strong motion intensity, respectively. The position of the letter is determined by the value of the original multi-mode fusion vector data through the dimensionality reduction.

The first three graphs separately represent the experimental data of each subject, and the fourth graph combines the data of three subjects and then draws them by the intensity classification. Analysing the position of the multi-mode vector data after the dimensionality reduction shows that the distribution of the individual multi-mode fusion vectors has a high degree of discrimination. The same letters are clustered together, and different letters are far apart. The result of the dimensionality reduction for the classification is more extreme.

In the first two graphs, the classifications of the letter $S$ and the letter $\mathrm{M}$ is more cross-over, which can also support the source of error between strong motion intensity and medium motion intensity in the confusion matrix. Compared with the first three figures, the mixed fourth figure shows that the distribution of the different letters is relatively scattered. The same letter logos converge into clusters, and more logos converge into multiple coherent blocks. On the whole, the model still shows a certain degree of discrimination for data with large individual differences. The results verify that the method of adding kinematic eigenvalues to the multi-mode fusion vector can alleviate the negative impact of the ECG signal due to individual differences to a certain extent.

\subsection{Analysis of adaptive trajectory optimization algorithm}

ATO(adaptive trajectory optimization)-DQN algorithm emphasizes on dynamic state interaction. In order to improve the processing speed of the algorithm, the offline data pretraining of Evaluating Q Network is carried out. The ratio of offline and online samples with motion intensity mutation labels is 1:5, and the feedback angle information recorded in the pre-training process is put into experience replay.

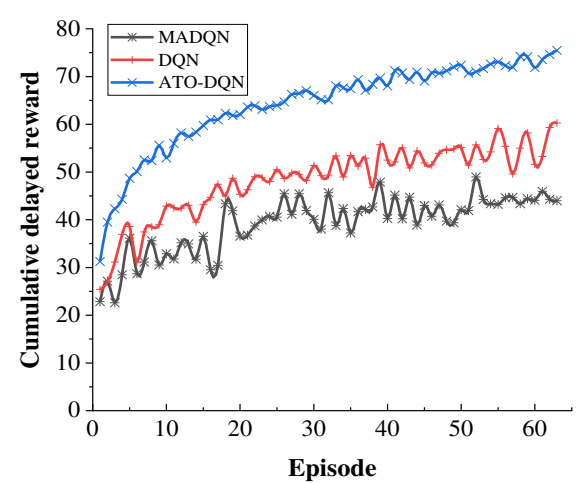

Figure.18 Comparison of cumulative delayed reward for MADQN, traditional DQN and ATO-DQN

The cumulative delayed reward for MADQN (Multi-agent DQN), traditional DQN and ATO-DQN under motion intensity mutation state as the episode increases is shown in Fig.18. Attributed to the pre-training operation, the convergence speed and stability of ATO-DQN algorithm are significantly higher than those of MADQN and DQN. More obviously, ATO-DQN algorithm obtains higher cumulative delayed reward. In contrast, MADQN is more suitable for multi-agent (at least more than two agents) cooperative control. 


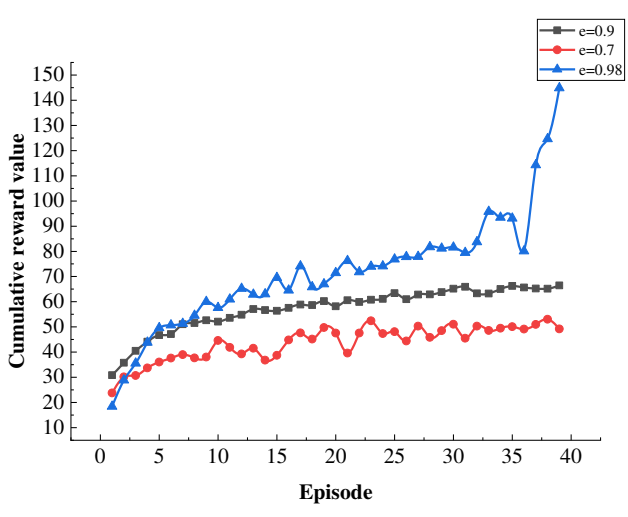

Figure.19 Performance of reward value and convergence rate under different learning rates. When the learning rate $e$ is 0.7 , the model step length is too short and the convergence speed is too slow; When the learning rate is 0.98 , the convergence speed is greatly improved but can not converge.

Figure.19 shows the performance of reward value and convergence rate under different learning rates. For each motion cycle, 400 groups of samples can be obtained and the learning rate $(e=0.9)$ is used to accelerate the iteration. Then, the action output is randomly selected by the $\varepsilon$-greed algorithm, and the stability is high under multiple tests.

The rehabilitation motion trajectory used in the perception model validation experiment is imported into ATO-DQN algorithm model as the test datasets, and the real-time motion intensity is set as the mixed state of "Strong" and "Weak", and the trajectory control signal is outputted to the exoskeleton manipulator and executed without load. The actual motion trajectory is shown in Figure. 20(a), while the trajectory without algorithm optimization is shown in Figure. 20(b).

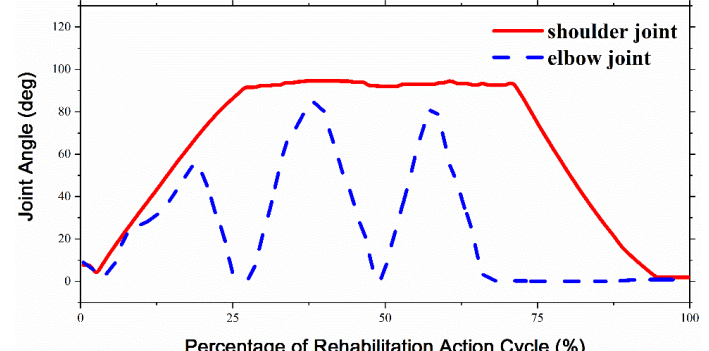

Percentage of Rehabilitation Action Cycle (\%)
(a). Rehabilitation motion trajectory without ATO-DQN algorithm

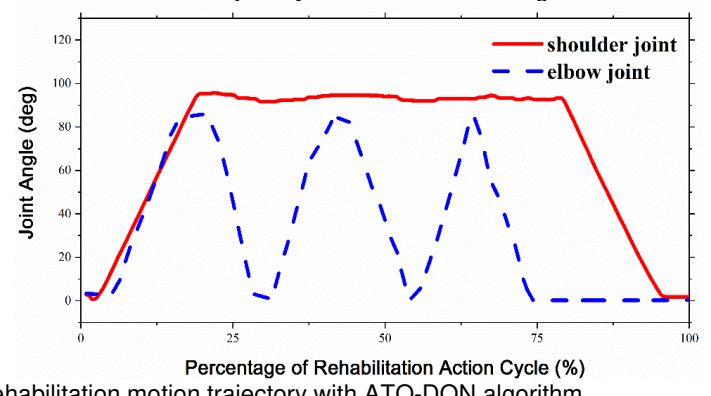

(b). Rehabilitation motion trajectory with ATO-DQN algorithm

Figure.20 Comparison of the trajectories of two states

It can be seen from Figure.20 that the output speed of the control method without ATO-DQN algorithm is relatively unstable in the face of continuous motion state mutation, resulting in the distortion of the actual output trajectory. The trajectory of elbow and shoulder joint begins to jitter and deform between 0 20\% cycles (Corresponding to the first two stages in Table 6, namely the transition stage from "strong" to "moderate" state).

In contrast, after the optimization of ATO-DQN algorithm, due to the action selection refinement and dynamic interaction ability, the tracking effect of motion trajectory on real-time environmental changes is enhanced, and the overall fluctuation is small, which can better guarantee the motion safety of patients.

\section{Conclusions}

In this paper, the time domain, the frequency domain eigenvalue of ECG and the angular velocity deviation of the encoder were extracted as the input layer of the motion intensity variation interval estimation model, which was constructed for classification and identification. In the experiment, the results verify that the method of adding kinematic eigenvalues to the multi-mode fusion vector can alleviate the negative impact of the ECG signal due to individual differences to a certain extent. When the proposed method comparing with those conventional image classification frameworks, the structure optimization of our improved inception-CNN network plays a crucial role in reducing redundant network parameters and improving network running time. After the optimization of ATO-DQN algorithm, the tracking effect of motion trajectory on real-time environmental changes enhance, which can better guarantee the motion safety of patients.

Our work also has many limitations. Compared with other methods, the precision, recall and F1 index have not been significantly improved. In Analysis of adaptive trajectory optimization algorithm, the types and evaluation indicators of DQN methods are not comprehensive enough. Most importantly, the current technology does not have good versatility in the application of manipulators, only in the angular velocity sensor and ECG signal multimodal feature vector shows good result. In subsequent research, our work can refine the classification limit of motion intensity and improve the speed correction accuracy of trajectory. We hope the trajectory correction technology will develop towards the direction of generalization and diversification.

\section{Abbreviations}

ECG: Electrocardiogram; MIVIE: Motion Intensity Variation Interval Estimation; ATO-DQN: Adaptive Trajectory Optimization-Deep Q Network; MADQN: Multi-agent DQN; CNN: Convolutional Neural Network; ACR: average convergence rate

\section{Acknowledgements}

We thank the participants in this study for their willingness to devote energy and sweat to completing the research work of this project.

\section{Contributions}

W.W. implemented and performed the experiments, analyzed the data and wrote the manuscript. H.L. performed the experiments, analyzed the data and wrote the manuscript, C.Z. conceived the experiment, analyzed the data and critically revised the manuscript. D.K. performed the experiments and critically revised the manuscript, P.Z. conceived the experiment and critically revised the manuscript.

\section{Funding}

This work is supported by the Natural Science Foundation of Shaanxi Province (Grant No. 2020JM-131 and 2020KW058), the Guangdong Basic and Applied Basic Research 
Foundation (2019A1515111176), and the Guangdong Science and Technology Innovation Strategy Special Foundation (2019B090904007).

\section{Ethics approval and consent to participate}

The data acquisition protocol was approved by Medical and Experimental Animal Ethics Committee of Northwestern Polytechnical University, Xi'an, China (approbation number: 6101030222595-202001001).

\section{Competing in interests}

The author(s) declare(s) that they have no competing interests.

\section{References}

[1] C. Fisahn, M. Aach, O. Jansen, M. Moisi, A. Mayadev, K. T. Pagarigan, J. R. Dettori, and T. A. Schildhauer, "The Effectiveness and Safety of Exoskeletons as Assistive and Rehabilitation Devices in the Treatment of Neurologic Gait Disorders in Patients with Spinal Cord Injury: A Systematic Review," Global Spine Journal, vol. 6, pp. 822-841, 2016.

[2] Y. Fan and Y. Yin, "Active and Progressive Exoskeleton Rehabilitation Using Multisource Information Fusion From EMG and Force-Position EPP," IEEE transactions on biomedical engineering, vol. 60, pp. 3314-3321, 2013.

[3] G. Chen, C. K. Chan, Z. Guo, and H. Yu, "A review of lower extremity assistive robotic exoskeletons in rehabilitation therapy," Critical reviews in biomedical engineering, vol. 41, p. 343, 2013.

[4] M. Dežman and A. Gams, "Rotatable cam-based variable-ratio lever compliant actuator for wearable devices," Mechanism and Machine Theory, vol. 130, pp. 508-522, 2018.

[5] S. U. Marasingha-Arachchige, J. Á. Rubio-Arias, P. E. Alcaraz, and L. H. Chung, "Factors that affect heart rate variability following acute resistance exercise: A systematic review and meta-analysis," Journal of Sport and Health Science, 2020.

[6] E. Xydas and L. S. Louca, "Planar conformity of movements in 3D reaching tasks for persons with Multiple Sclerosis," Human Movement Science, vol. 62, pp. 221-234, 2018.

[7] Z. Li, Z. Huang, W. He, and C. Su, "Adaptive Impedance Control for an Upper Limb Robotic Exoskeleton Using Biological Signals," IEEE Transactions on Industrial Electronics, vol. 64, pp. 1664-1674, 2017.

[8] E. A. Thomson, K. Nuss, A. Comstock, S. Reinwald, S. Blake, R. E. Pimentel, B. L. Tracy, and K. Li, "Heart rate measures from the Apple Watch, Fitbit Charge HR 2, and electrocardiogram across different exercise intensities," Journal of Sports Sciences, vol. 37, pp. 1411-1419, 2018.

[9] J. Sun, Z. Guo, D. Sun, S. He, and X. Xiao, "Design, modeling and control of a novel compact, energy-efficient, and rotational serial variable stiffness actuator (SVSA-II)," Mechanism and Machine Theory, vol. 130, pp. 123-136, 2018.

[10] N. Yozbatiran, J. Berliner, M. O Malley, A. Pehlivan, Z. Kadivar, C. Boake, and G. Francisco, "Robotic training and clinical assessment of upper extremity movements after spinal cord injury: A single case report," Journal of Rehabilitation Medicine, vol. 44, p. 186, 2012.

[11] F. Zyener, H. B. Rossiter, S. A. Ward, and B. J. Whipp, "Influence of exercise intensity on the on- and off-transient kinetics of pulmonary oxygen uptake in humans," Journal of Physiology, vol. 533, pp. 891-902, 2010

[12] Y. BIN and Q. JIANG, "Human Motion Intensity Detection Embedded in Holter," Chinese Journal of Medical Physics, p. 18, 2012.

[13] H. Lee, H. Chung, H. Ko, and J. Lee, "Wearable Multichannel Photoplethysmography Framework for Heart Rate Monitoring During Intensive Exercise," IEEE Sensors Journal, vol. 18, pp. 2983-2993, 2018.
[14] F. S. Lin and K. Dana, "Online Segmentation of Human Motion for Automated Rehabilitation Exercise Analysis," IEEE Transactions on Neural Systems and Rehabilitation Engineering, 2013.

[15] M. Längkvist, L. Karlsson and A. Loutfi, "A review of unsupervised feature learning and deep learning for time-series modeling," Pattern Recognition Letters, vol. 42, pp. 11-24, 2014.

[16] W. Wang, A. Tan and L. Teow, "Semantic memory modeling and memory interaction in learning agents," IEEE Transactions on Systems, Man, and Cybernetics: Systems, vol. 47, pp. 2882-2895, 2016.

[17] G. Petmezas, K. Haris, L. Stefanopoulos, V. Kilintzis, A. Tzavelis, J. A. Rogers, A. K. Katsaggelos, and N. Maglaveras, "Automated Atrial Fibrillation Detection using a Hybrid CNNLSTM Network on Imbalanced ECG Datasets," Biomedical Signal Processing and Control, vol. 63, p. 102194, 2021.

[18] T. Karlsen, I. Aamot, M. Haykowsky, and Ø. Rognmo, "High Intensity Interval Training for Maximizing Health Outcomes," Progress in Cardiovascular Diseases, vol. 60, pp. 67-77, 2017.

[19] R. Bailon, N. Garatachea, I. de la Iglesia, J. A. Casajus, and P. Laguna, "Influence of Running Stride Frequency in Heart Rate Variability Analysis During Treadmill Exercise Testing," IEEE Transactions on Biomedical Engineering, vol. 60, pp. 1796-1805, 2013.

[20] J. Liu, H. Li, R. Wu, Q. Zhao, Y. Guo, and L. Chen, "A survey on deep learning methods for scene flow estimation," PATTERN RECOGNITION, vol. 106, 2020.

[21] J. Schmidhuber, "Deep learning in neural networks: An overview," Neural Networks, vol. 61, pp. 85-117, 2015.

[22] U. Cote-Allard, C. L. Fall, A. Drouin, A. Campeau-Lecours, C. Gosselin, K. Glette, F. Laviolette, and B. Gosselin, "Deep Learning for Electromyographic Hand Gesture Signal Classification Using Transfer Learning," IEEE transactions on neural systems and rehabilitation engineering, vol. 27, pp. 760-771, 2019.

[23] A. Bahador, M. Yousefi, M. Marashi, and O. Bahador, "High accurate lightweight deep learning method for gesture recognition based on surface electromyography," Computer Methods and Programs in Biomedicine, vol. 195, p. 105643, 2020.

[24] K. Baur, A. Schättin, E. D. de Bruin, R. Riener, J. E. Duarte, and P. Wolf, "Trends in robot-assisted and virtual reality-assisted neuromuscular therapy: a systematic review of health-related multiplayer games," Journal of neuroengineering and rehabilitation, vol. 15, p. 107, 2018.

[25] J. Fang, Z. Mu, Z. Xu, L. Xie, G. Yang, and Q. Zhang, "Models for temporal-spatial parameters in walking with cadence ratio as the independent variable," Medical \& biological engineering \& computing, vol. 57, pp. 877-886, 2019.

[26] R. S. Calabrò, M. Accorinti, B. Porcari, L. Carioti, L. Ciatto, L. Billeri, V. A. Andronaco, F. Galletti, S. Filoni, and A. Naro, "Does hand robotic rehabilitation improve motor function by rebalancing interhemispheric connectivity after chronic stroke? Encouraging data from a randomised-clinical-trial," Clinical Neurophysiology, vol. 130, pp. 767-780, 2019.

[27] B. Peerdeman, D. Boere, H. Witteveen, H. Hermens, S. Stramigioli, H. Rietman, P. Veltink, and S. Misra, "Myoelectric forearm prostheses: state of the art from a user-centered perspective.," Journal of Rehabilitation Research \& Development, vol. 48, 2011.

[28] Z. Comert, A. M. Boopathi, S. Velappan, Z. Yang, and A. F. Kocamaz, "The influences of different window functions and lengths on image-based time-frequency features of fetal heart rate signals," in 2018 26th Signal Processing and Communications Applications Conference (SIU), 2018, pp. 1-4.

[29] M. Wewege, R. van den Berg, R. E. Ward, and A. Keech, "The effects of high-intensity interval training vs. moderate-intensity continuous training on body composition in overweight and obese adults: a systematic review and meta-analysis," Obesity Reviews, vol. 18, pp. 635-646, 2017.

[30] K. Ranjeet, A. Kuamr and R. K. Pandey, "ECG signal compression using optimum wavelet filter bank based on Kaiser 
[31] S. Cuomo, G. De Pietro, R. Farina, A. Galletti, and G. Sannino, "A revised scheme for real time ecg signal denoising based on recursive filtering," Biomedical Signal Processing and Control, vol. 27, pp. 134-144, 2016.

[32] R. R. Sharma and R. B. Pachori, "Baseline wander and power line interference removal from ECG signals using eigenvalue decomposition," Biomedical Signal Processing and Control, vol. 45, pp. 33-49, 2018.

[33] Y. Xiang, Z. Lin and J. Meng, "Automatic QRS complex detection using two-level convolutional neural network," Biomedical engineering online, vol. 17, pp. 1-17, 2018.

[34] S. A. Murray, K. H. Ha, C. Hartigan, and M. Goldfarb, "An assistive control approach for a lower-limb exoskeleton to facilitate recovery of walking following stroke," IEEE Transactions on Neural Systems and Rehabilitation Engineering, vol. 23, pp. 441449, 2014.

[35] G. Budzik and P. Turek, "Improved accuracy of mandible geometry reconstruction at the stage of data processing and modeling," AUSTRALASIAN PHYSICAL I\& ENGINEERING SCIENCES IN MEDICINE, vol. 41, pp. 687-695, 2018.

[36] N. Srivastava, G. Hinton, A. Krizhevsky, I. Sutskever, and R. Salakhutdinov, "Dropout: a simple way to prevent neural networks from overfitting," The journal of machine learning research, vol. 15, pp. 1929-1958, 2014.

[37] C. Szegedy, V. Vanhoucke, S. Ioffe, J. Shlens, and Z. Wojna, "Rethinking the Inception Architecture for Computer Vision,", 2016, pp. 2818-2826.

[38] X. Wu, Z. Li, Z. Kan, and H. Gao, "Reference Trajectory Reshaping Optimization and Control of Robotic Exoskeletons for Human-Robot Co-Manipulation," IEEE Transactions on Cybernetics, vol. 50, pp. 3740-3751, 2020.

[39] C. Zhang, X. Pan, H. Li, A. Gardiner, I. Sargent, J. Hare, and P. M. Atkinson, "A hybrid MLP-CNN classifier for very fine resolution remotely sensed image classification," ISPRS Journal of Photogrammetry and Remote Sensing, vol. 140, pp. 133-144, 2018.

[40] T. J. Jun, H. M. Nguyen, D. Kang, D. Kim, D. Kim, and Y. Kim, "ECG arrhythmia classification using a 2-D convolutional neural network," arXiv preprint arXiv:1804.06812, 2018.

[41] K. Simonyan and A. Zisserman, "Very Deep Convolutional Networks for Large-Scale Image Recognition," arXiv preprint arXiv:1409.1556, 2014

[42] P. Rajpurkar, A. Y. Hannun, M. Haghpanahi, C. Bourn, and A. Y. Ng, "Cardiologist-Level Arrhythmia Detection with Convolutional Neural Networks," arXiv preprint arXiv:1707.01836, 2017. 
Figures

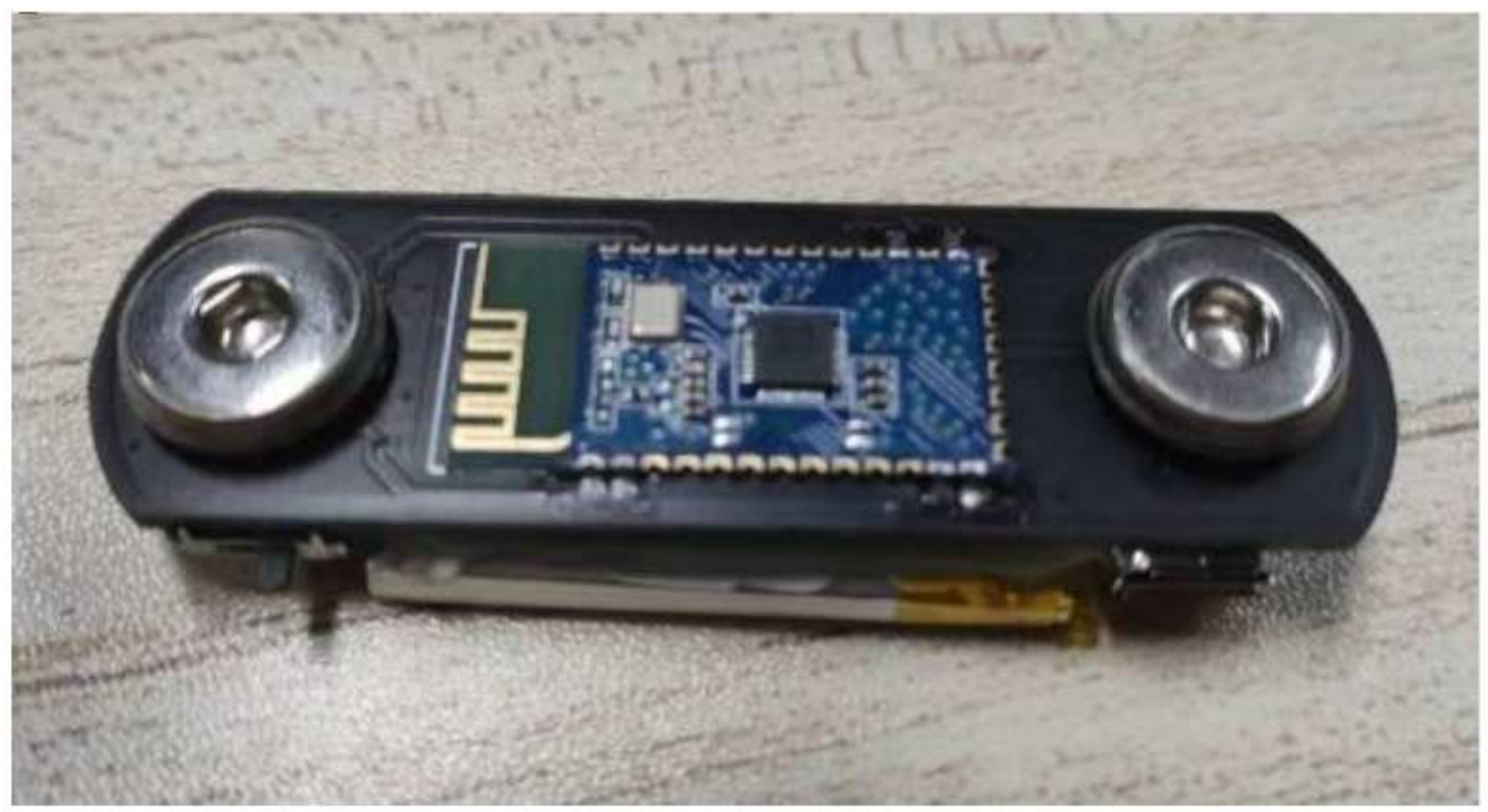

(a) BMD101 Bluetooth ECG sensor

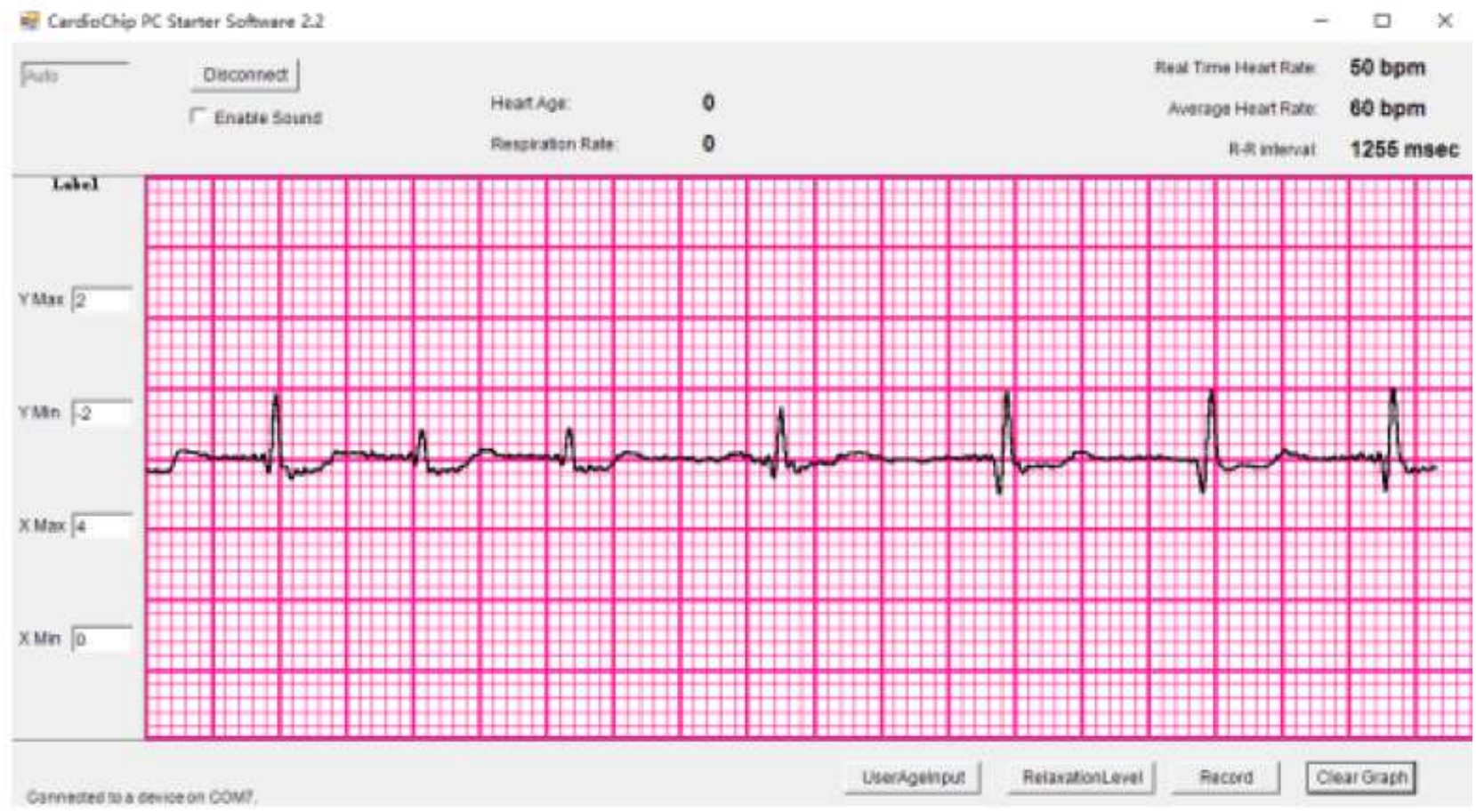

(b) Online ECG waveform display interface

Figure 1

BMD101 Bluetooth ECG acquisition module 


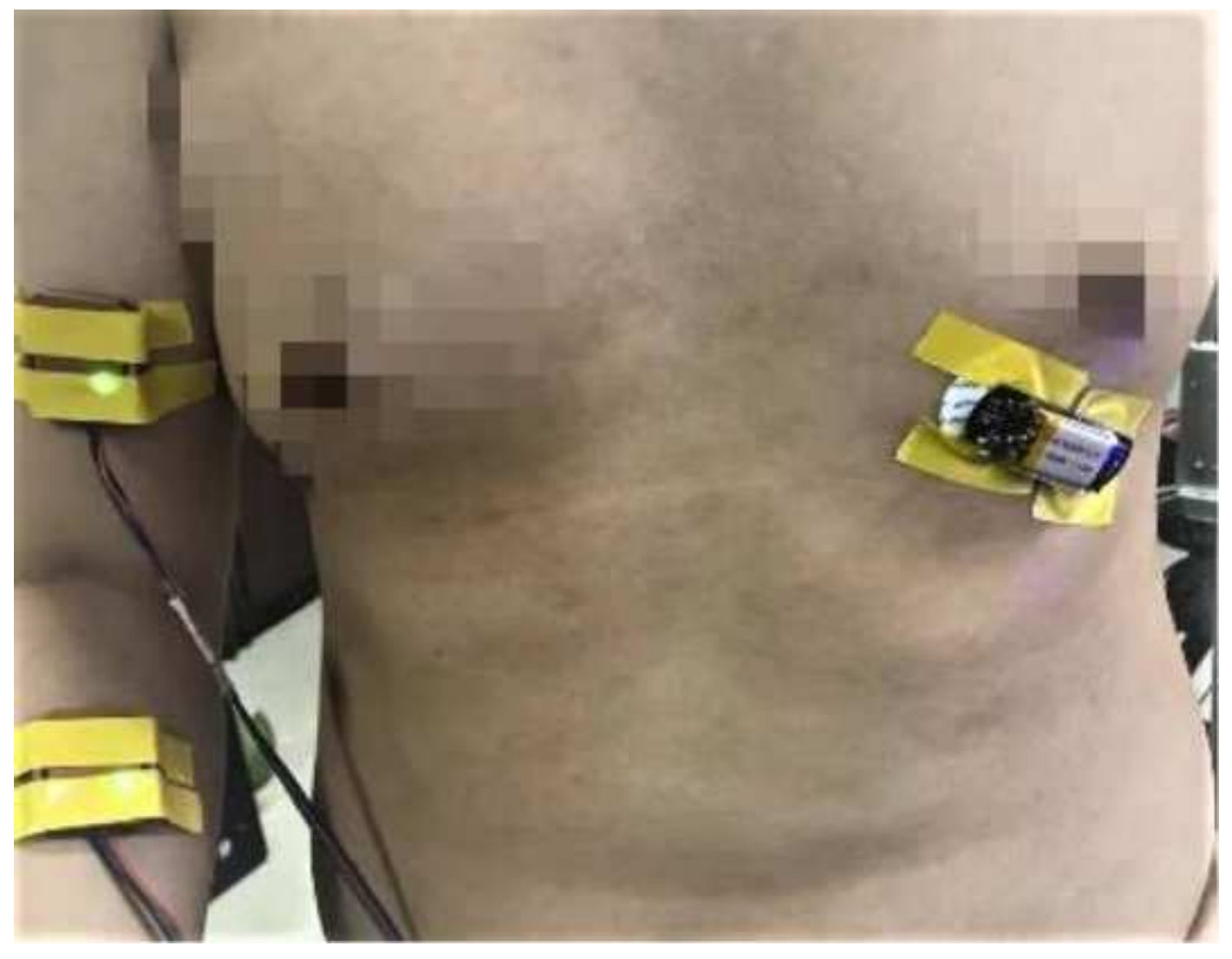

Figure 2

Rehabilitation scenario 


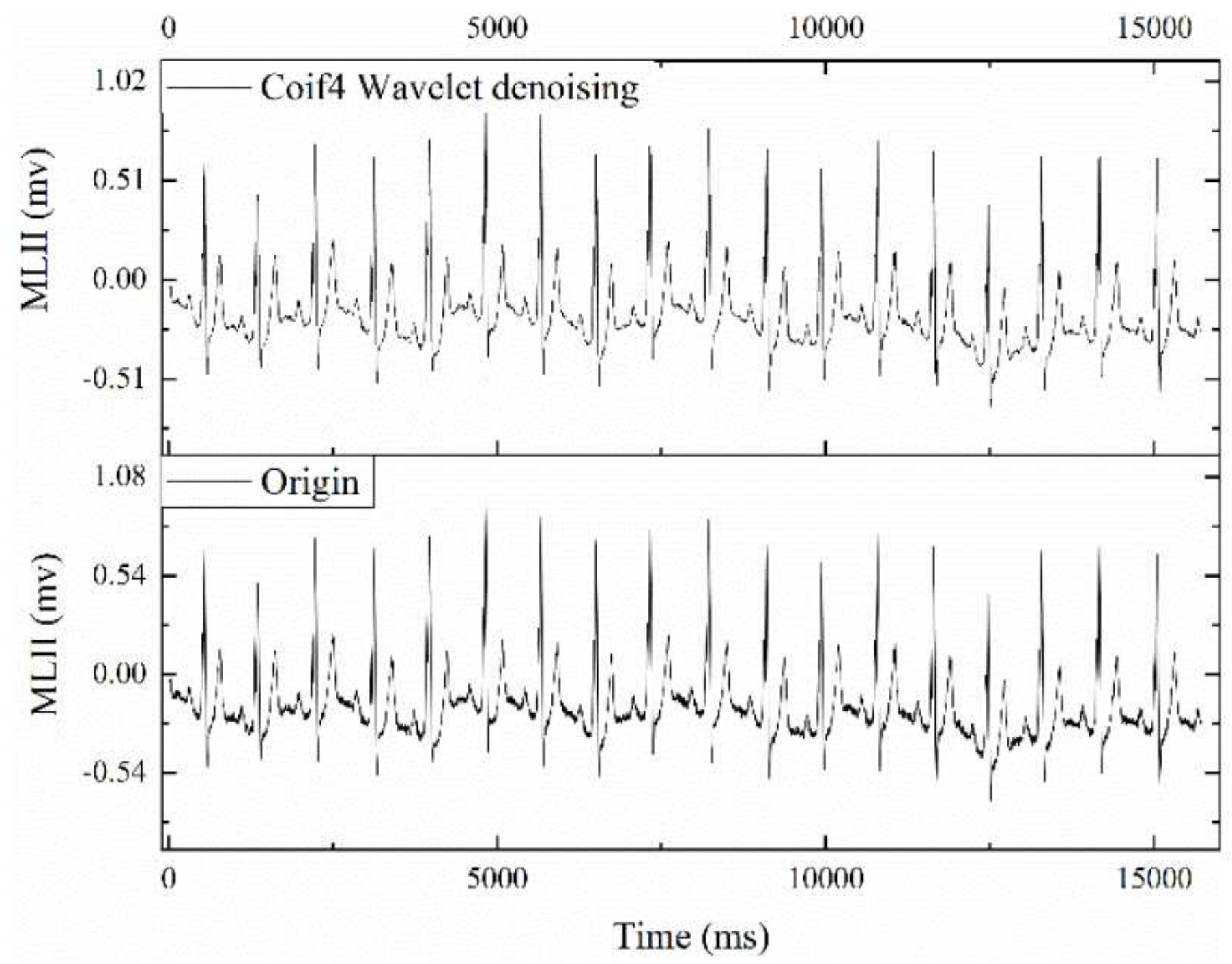

Figure 3

Comparison of denoising effect of improved Coif4 wavelet threshold algorithm and original signal 


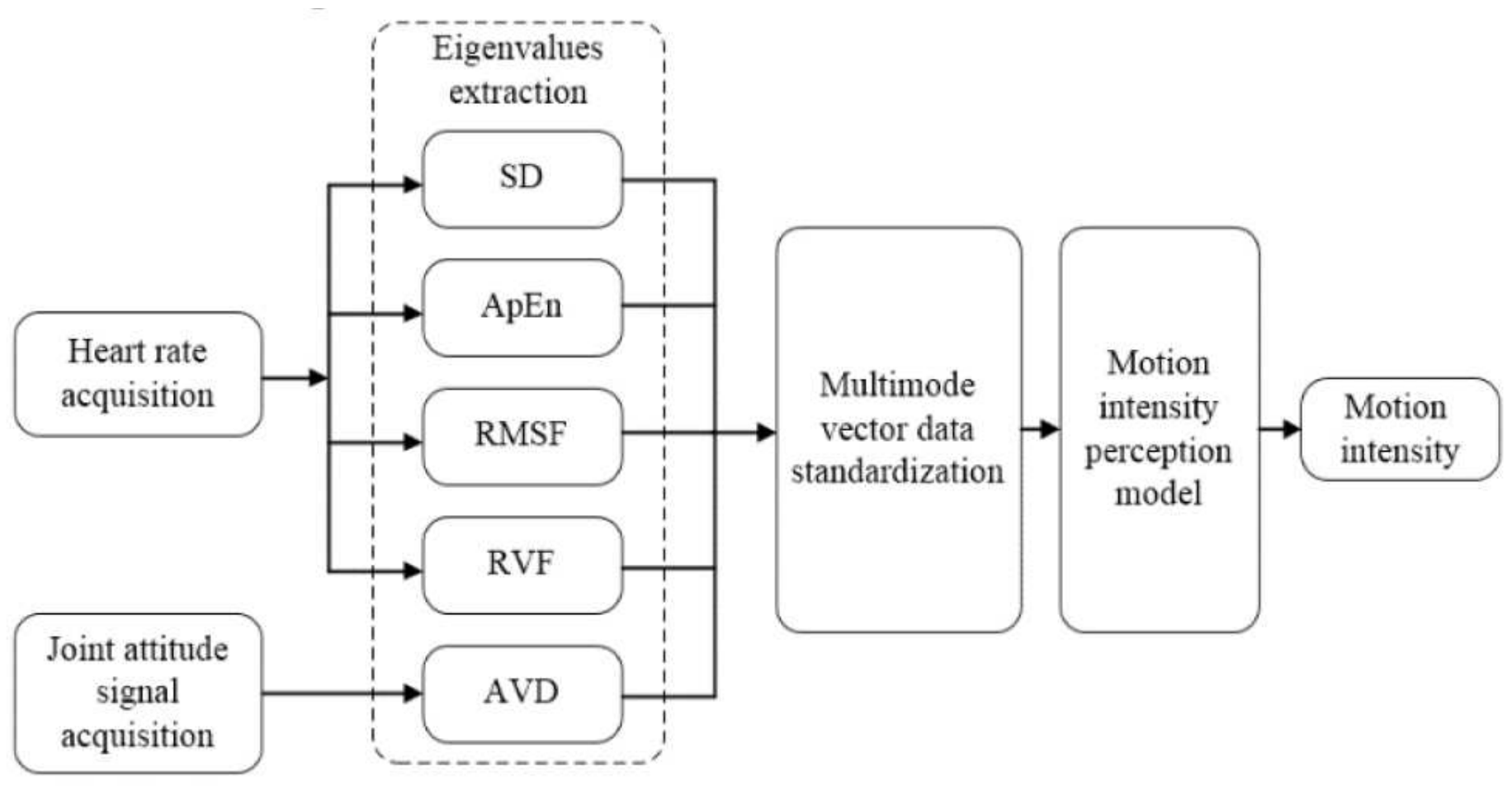

Figure 4

Multimode vector diagram

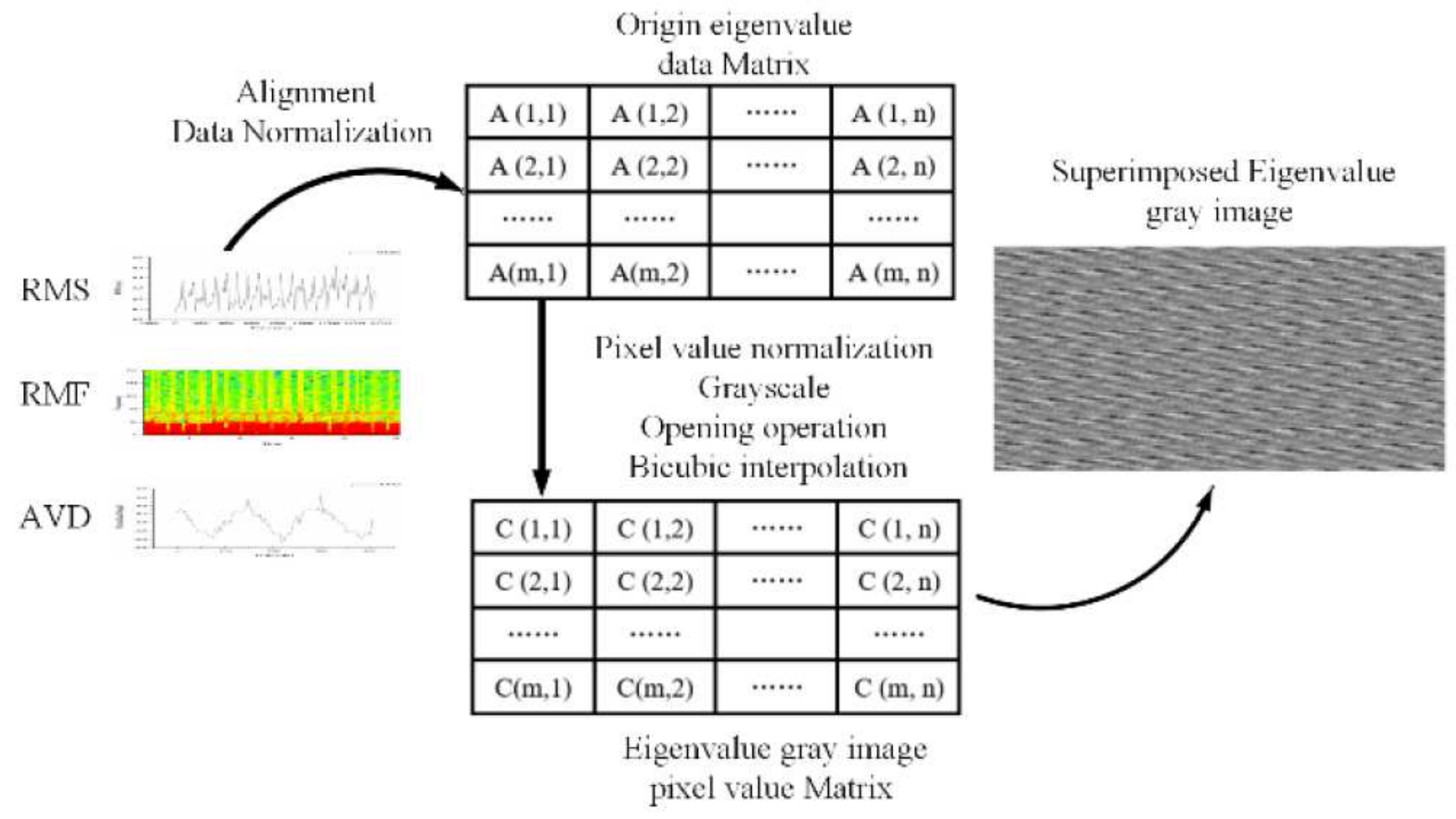

Figure 5

The conversion process from eigenvalue data matrix to gray pixel matrix 


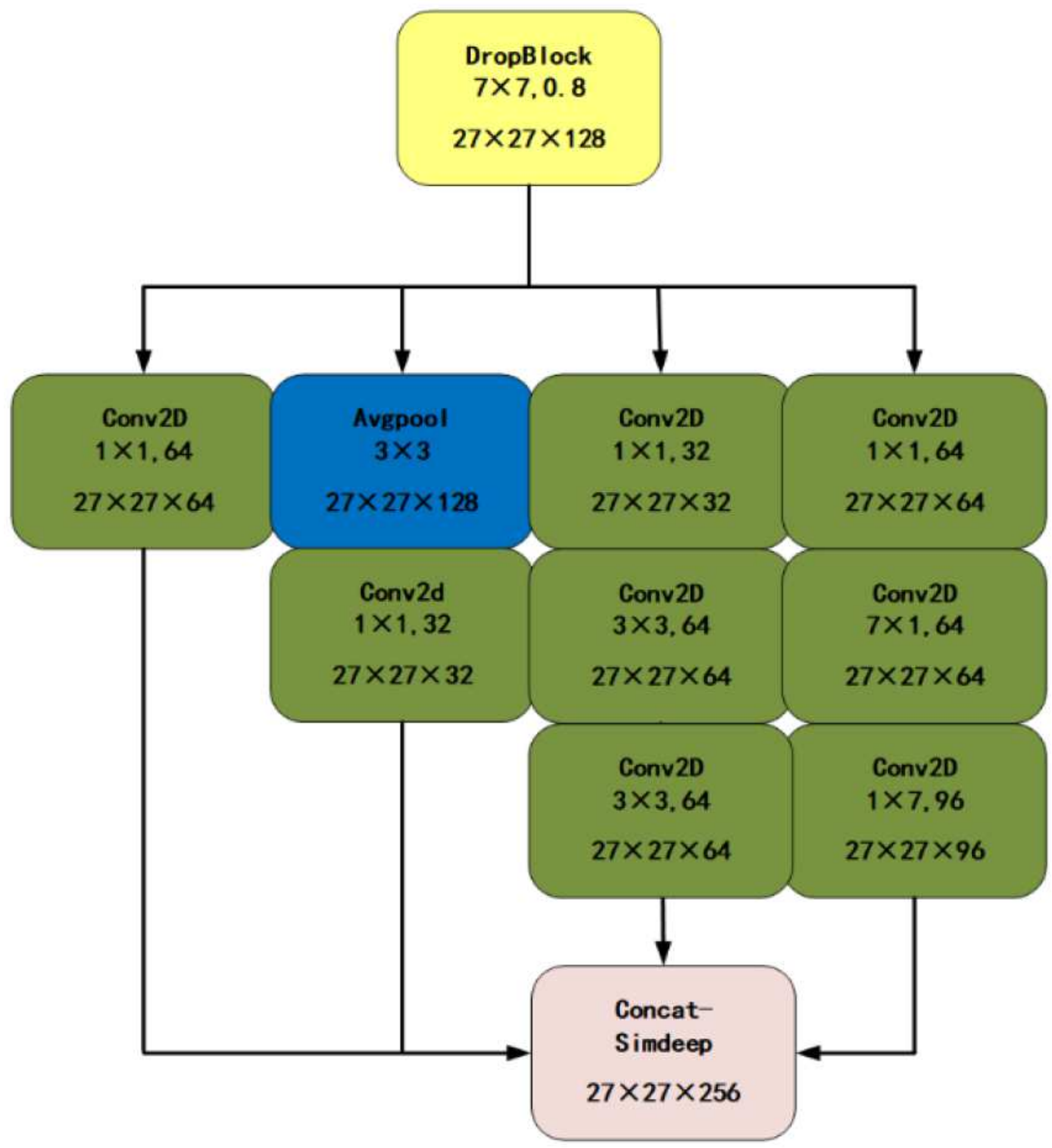

Figure 6

Inception-Simin module: From left to right, the third sub-column represents mini module of the " 5 th layer" module, the fourth sub-column represents mini module of "6th layer". 


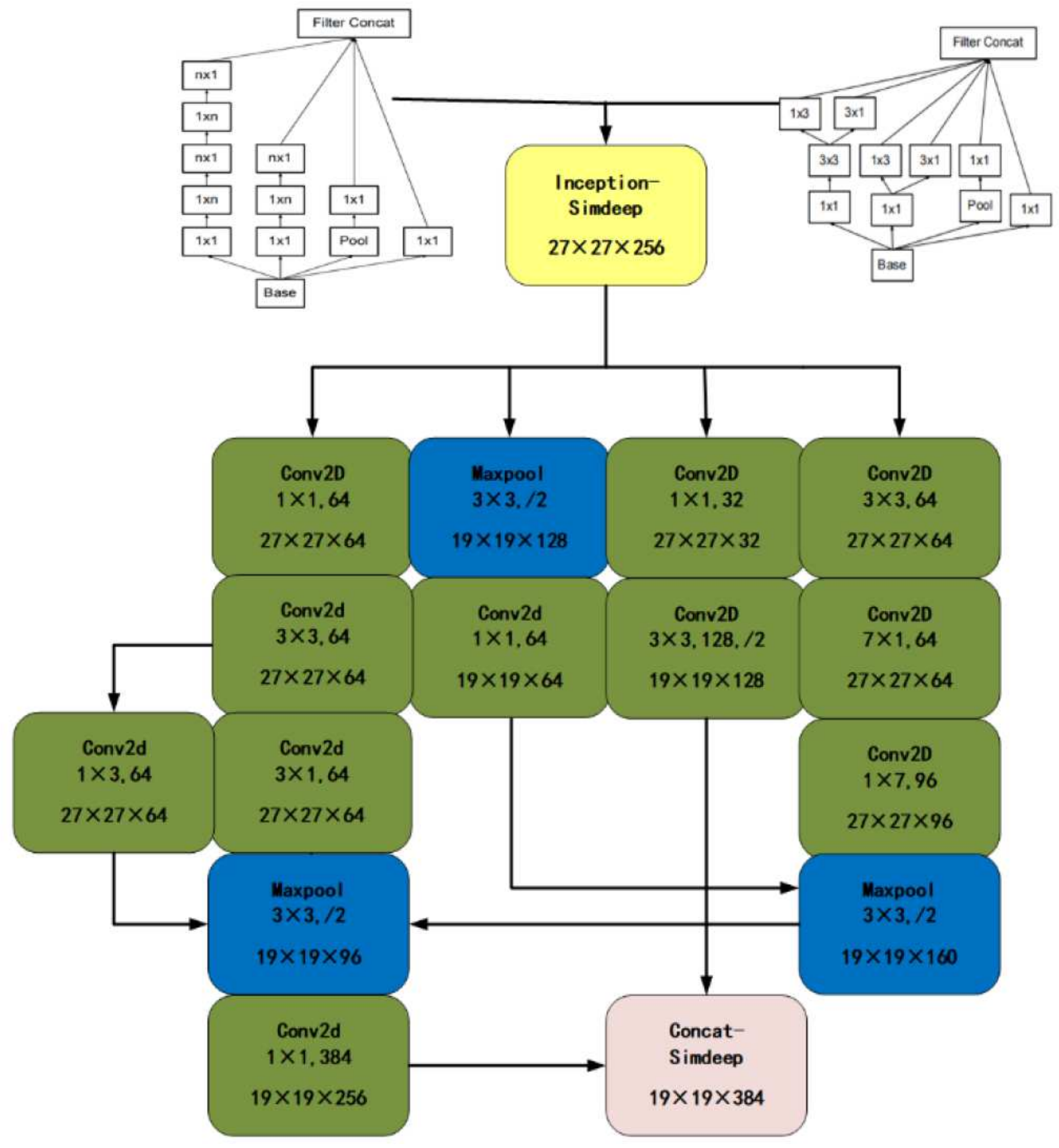

Figure 7

Inception-Simdeep module区" $3 \times 3$ " represents the size of kernel," $/ 2$ " represents stride of $2, " 64$ " represents the amount of kernel 


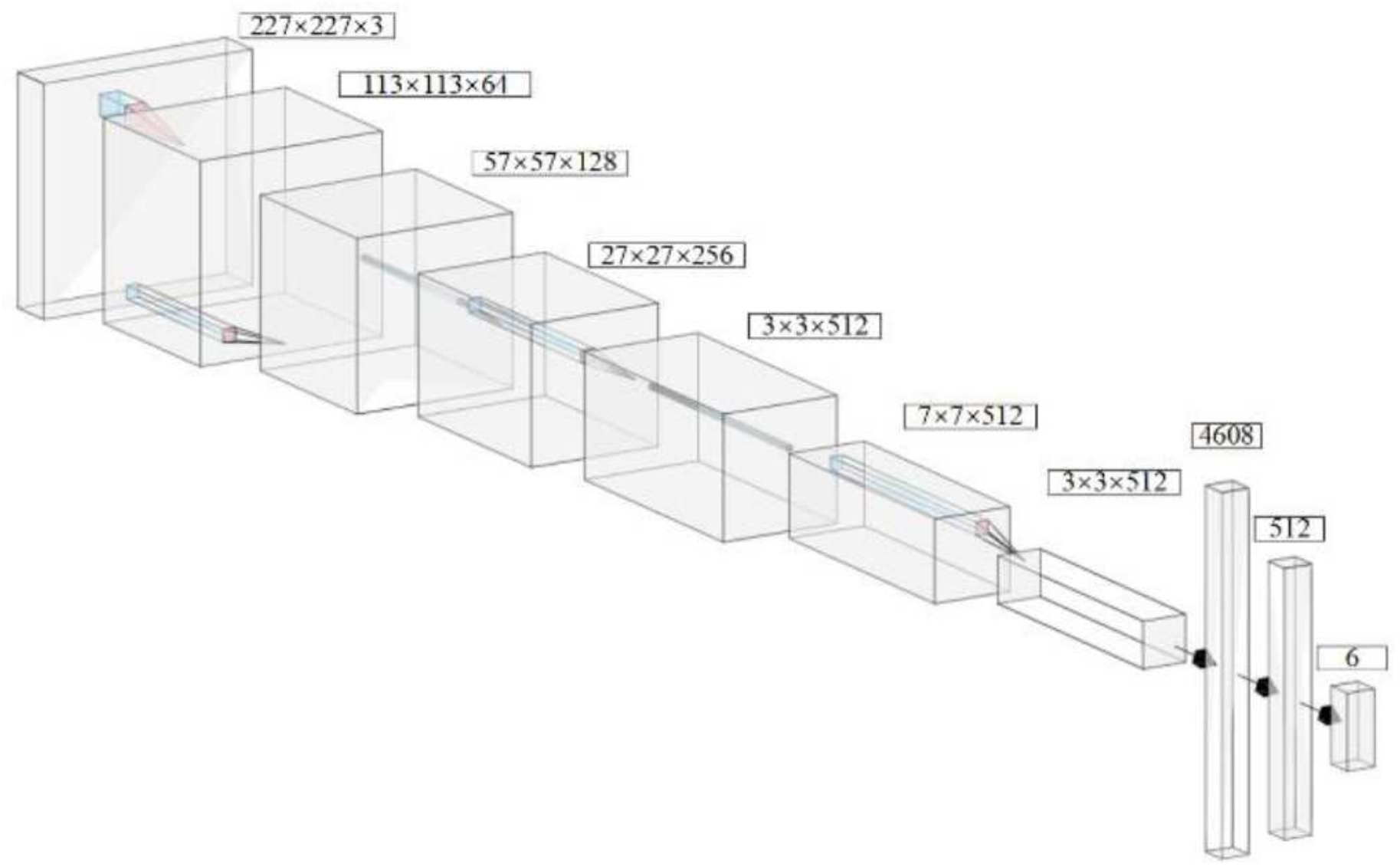

Figure 8

Motion intensity mutation perception model's network structure

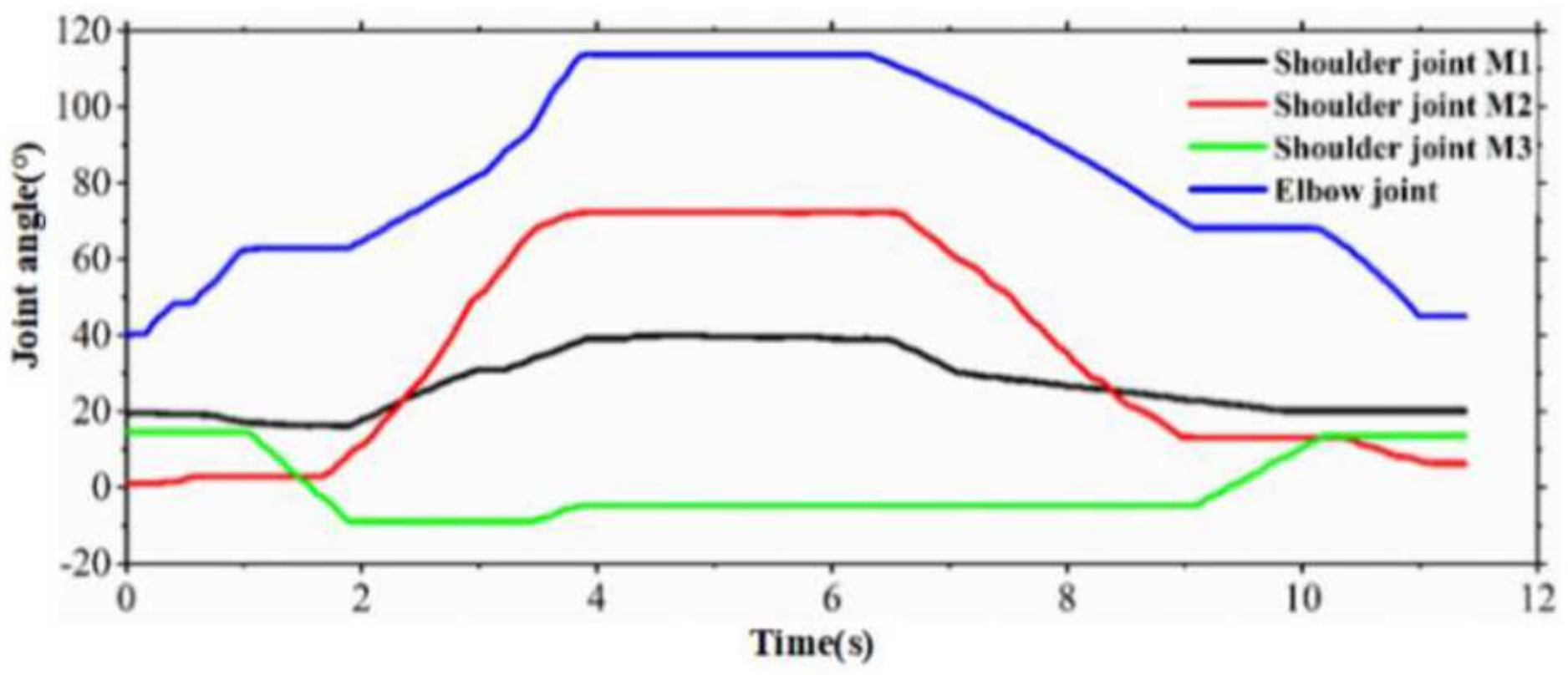

Figure 9

Motion trajectory of the shoulder and elbow joint angle 


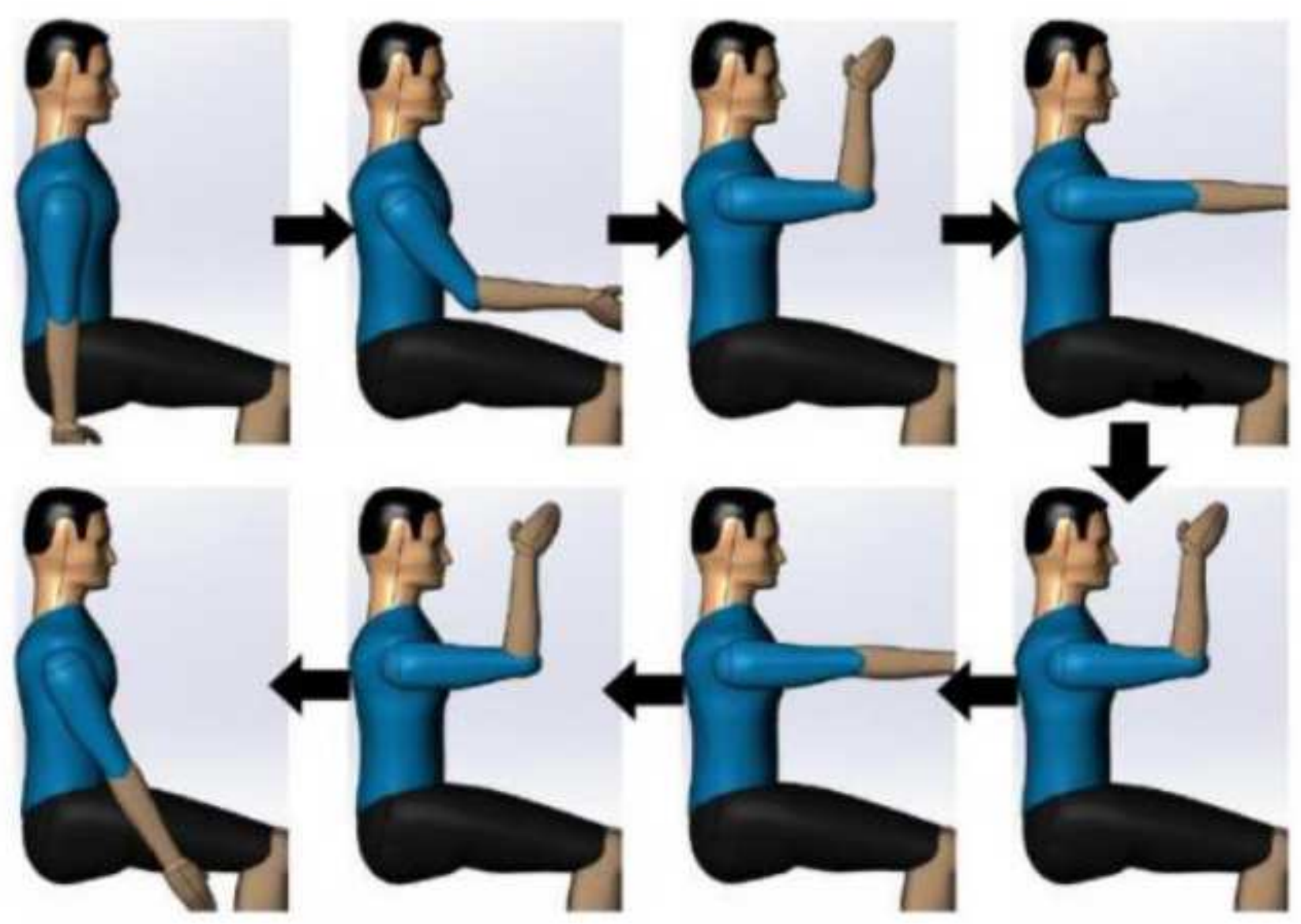

Figure 10

Rehabilitation motion decomposition

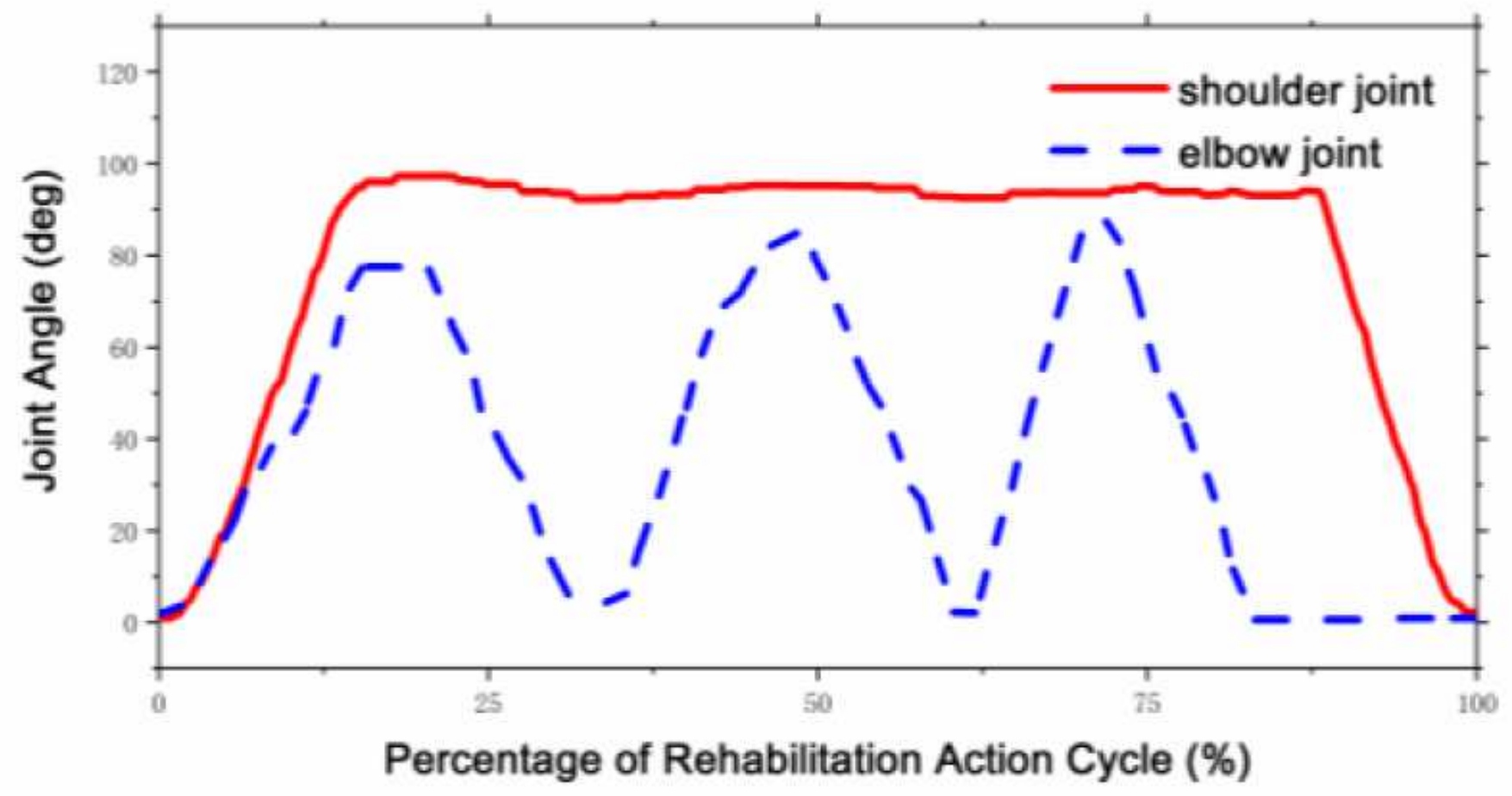




\section{Figure 11}

Rehabilitation motion trajectory through the demonstration teaching mode

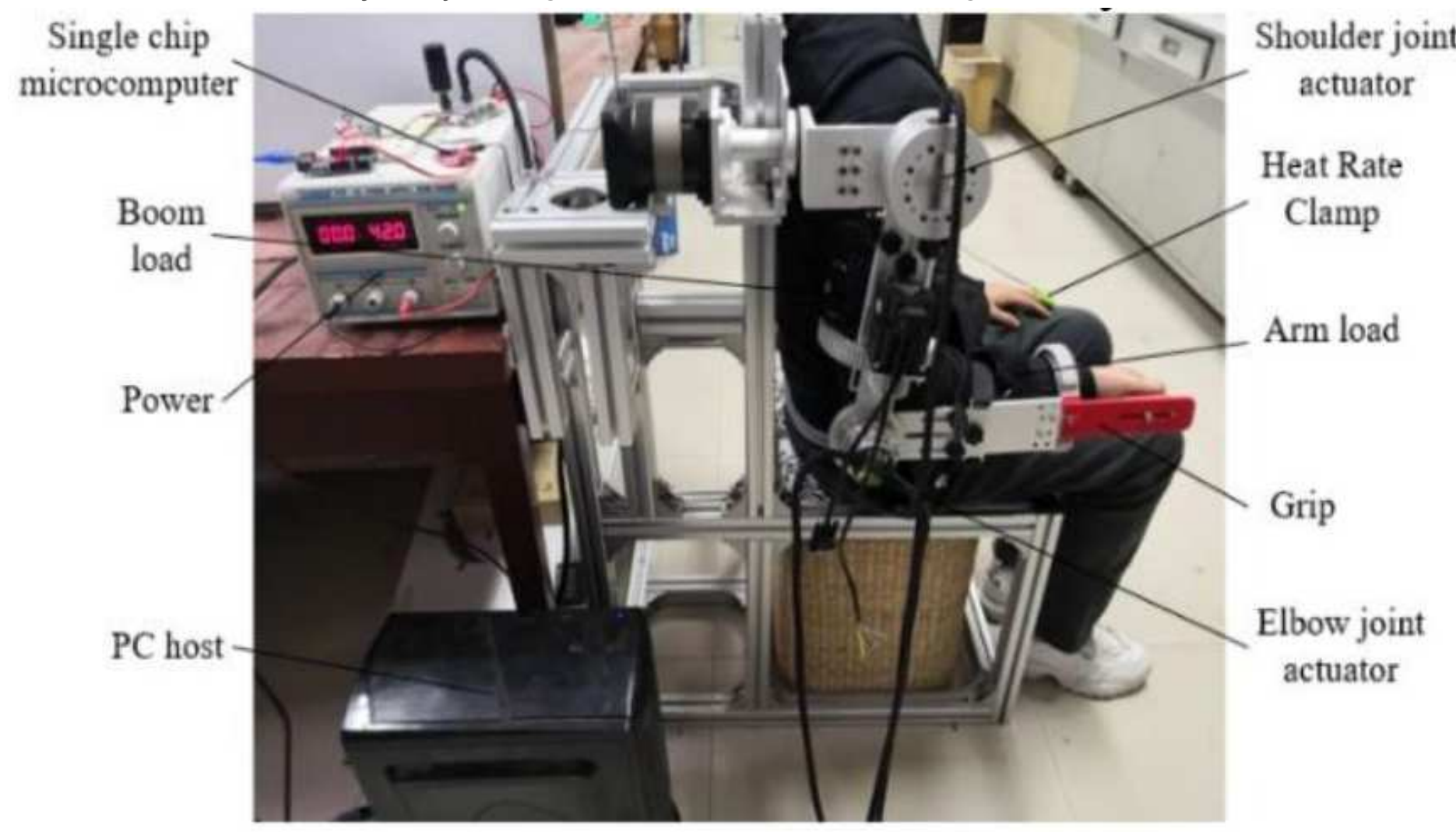

Figure 12

Rehabilitation motion experimental platform

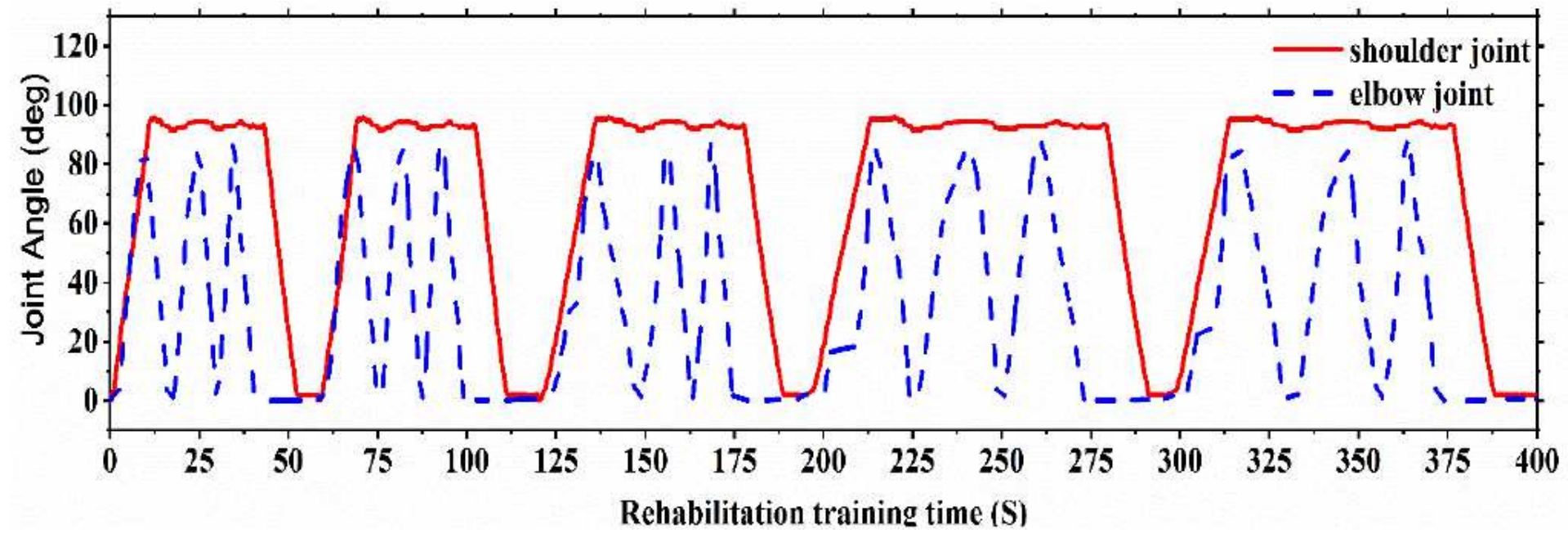

Figure 13

Experimental motion trajectory of five consecutive cycles 
Parallel coordinates plot with scaling of Standardization

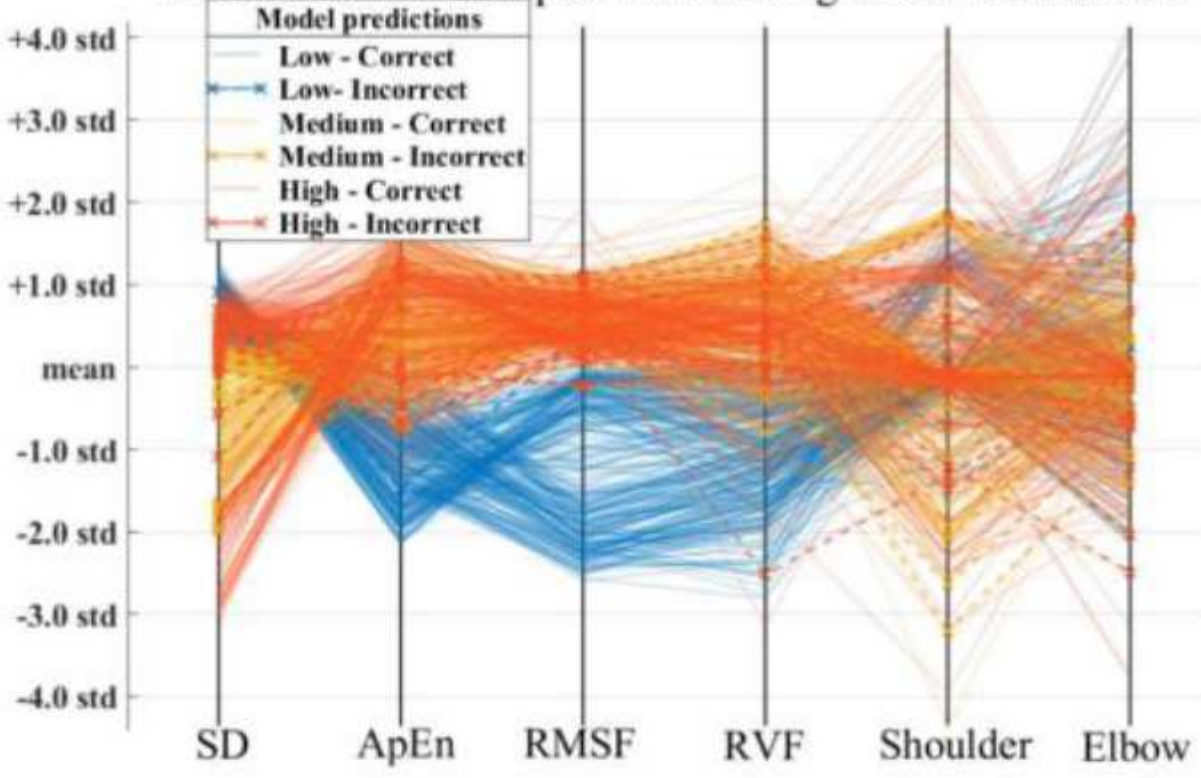

(a). Parallel coordinates plot with scaling of Standardization

Parallel coordinates plot with scaling of Normalization

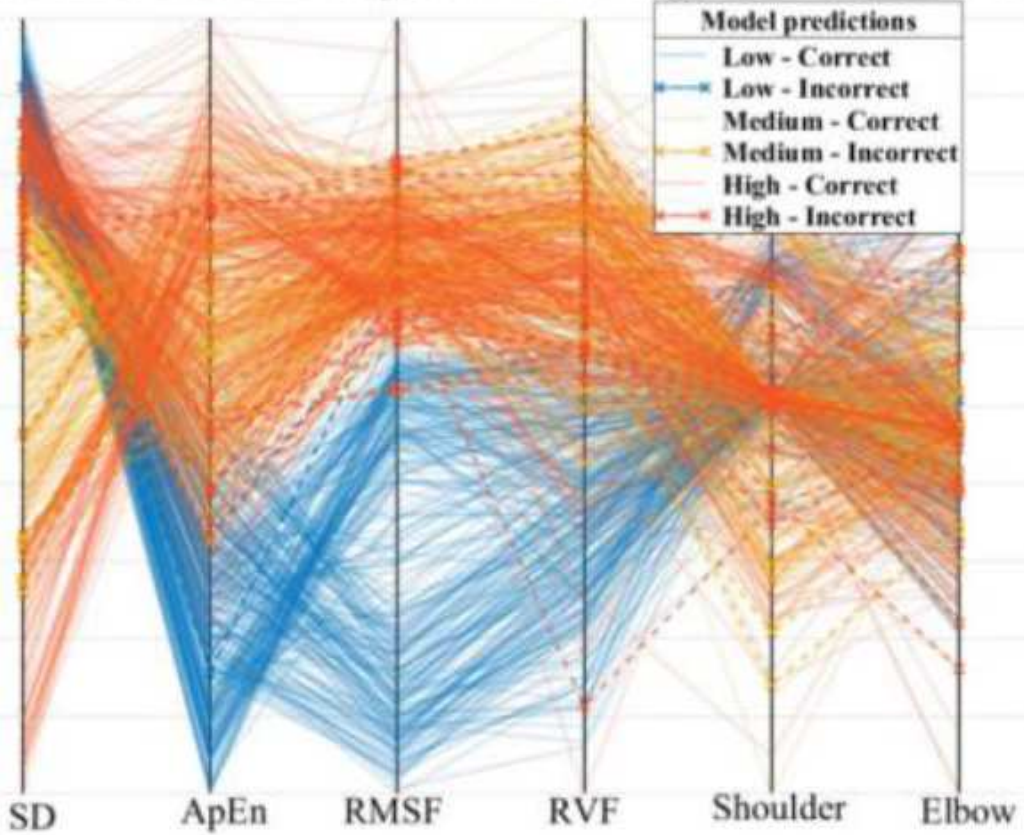

(b). Parallel coordinates plot with scaling of Normalization

Figure 14

Parallel coordinates plot with scaling of Standardization and Normalization 


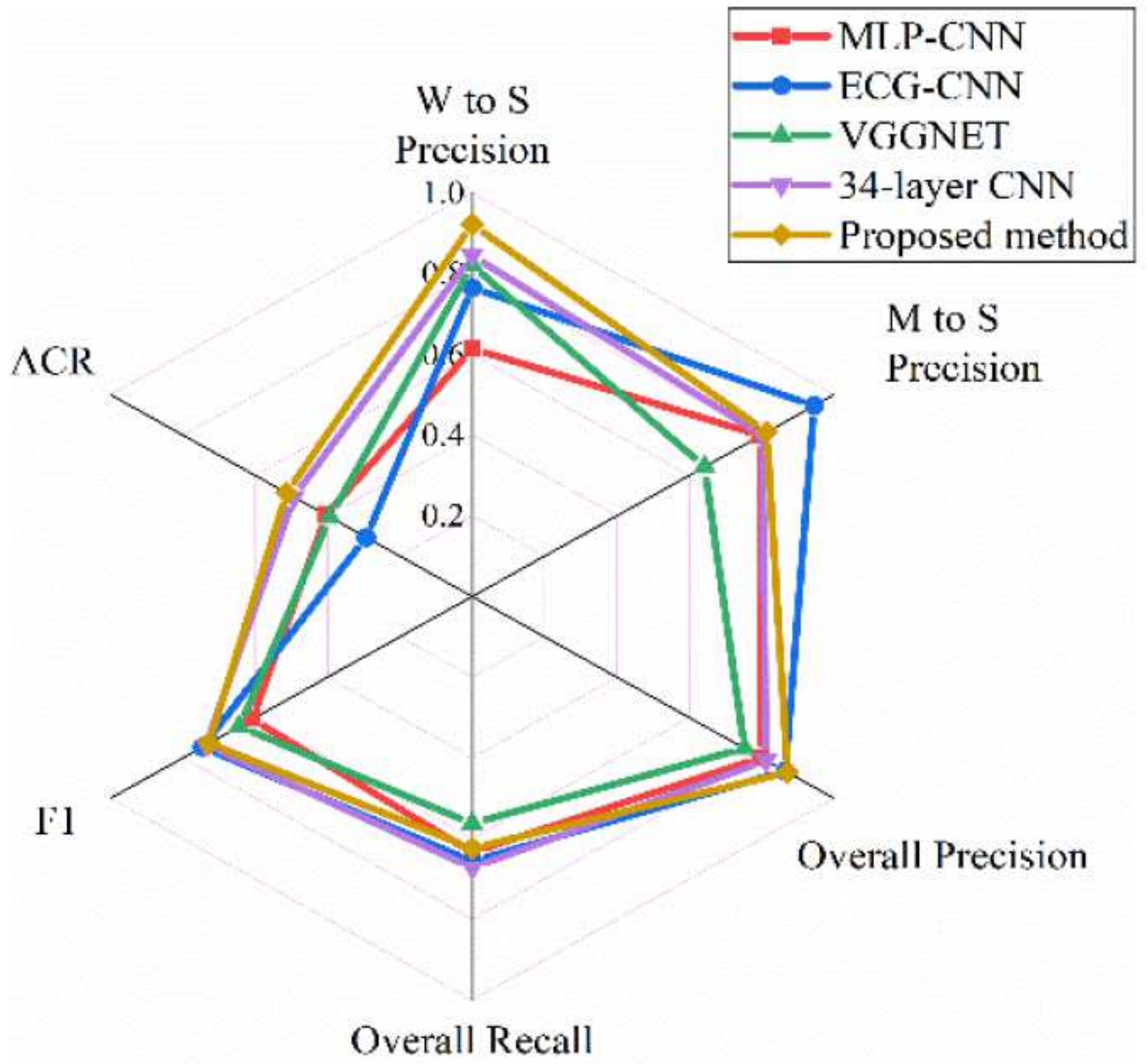

Figure 15

Radar chart of classification effect of CDB dataset 


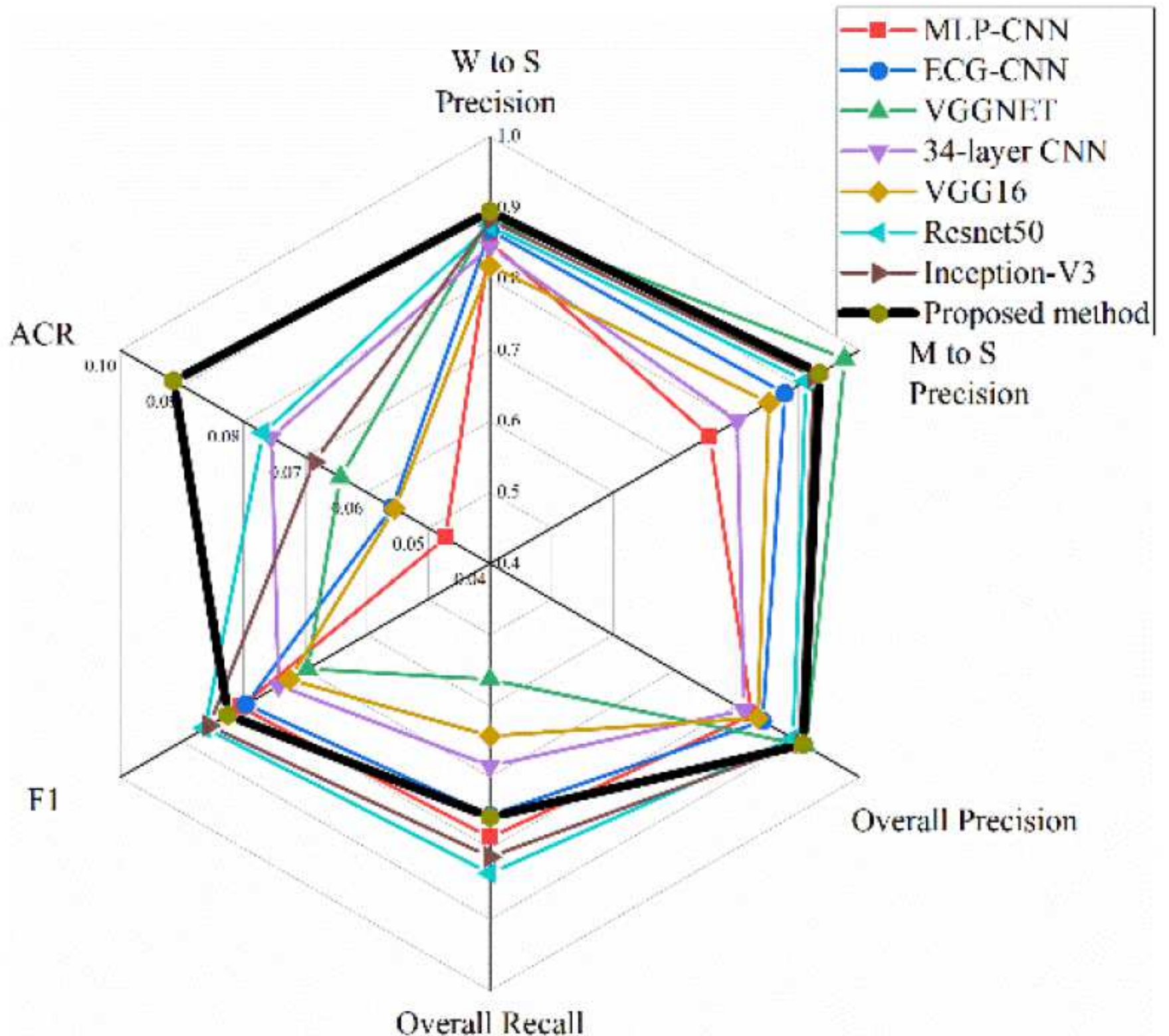

Figure 16

Radar Chart of Classification Effect of MIVIE Dataset 
Multimode feature vectors from the first subject

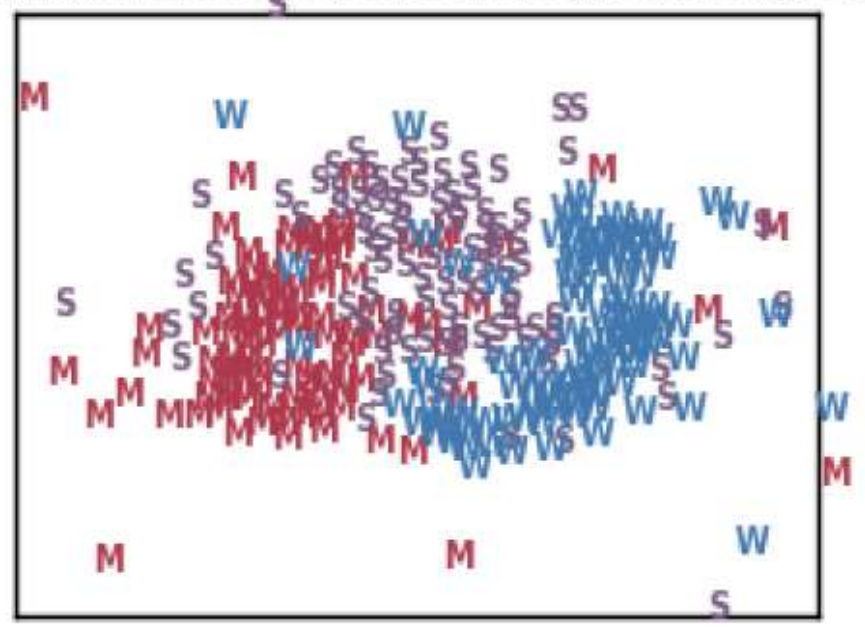

Multimode feature vectors from the third subject

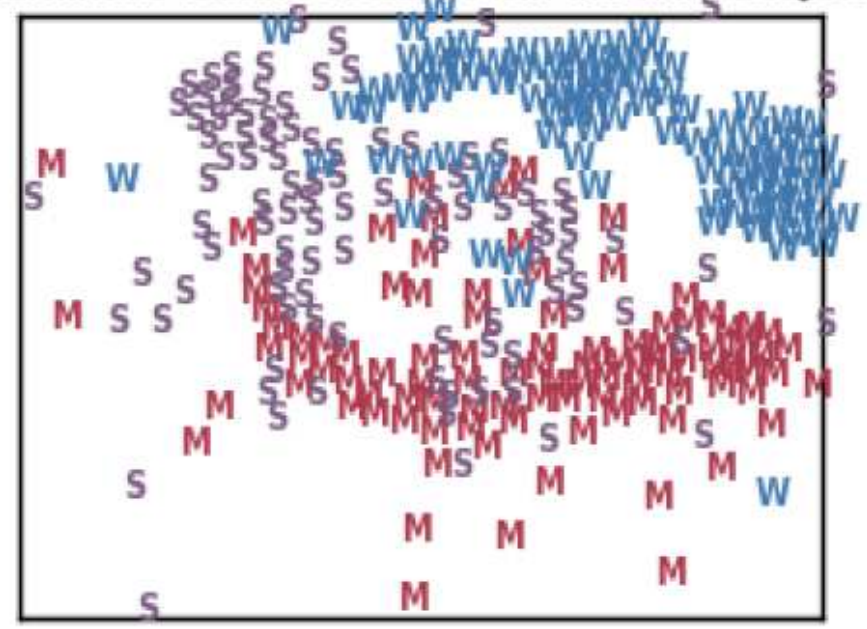

Multimode feature vectors from the second subject

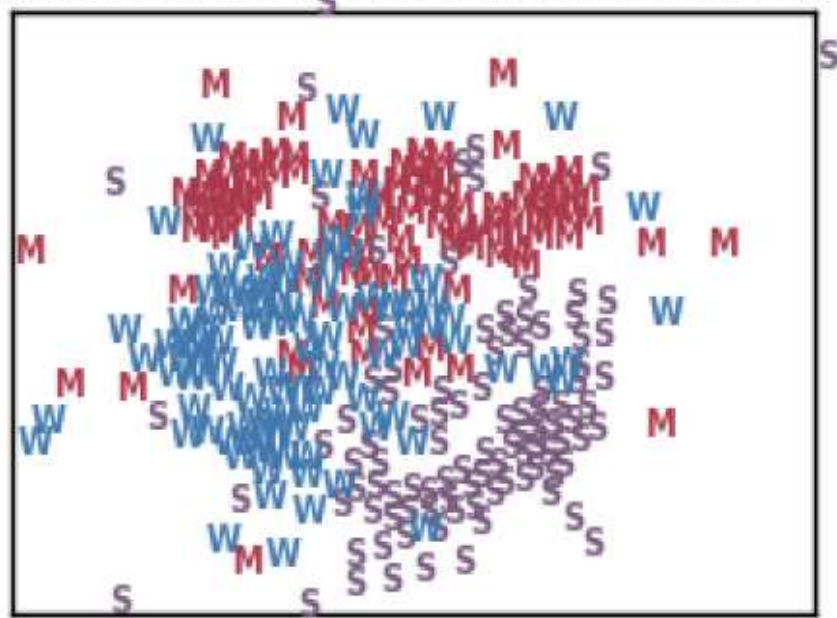

Multimode feature vectors from three subjects

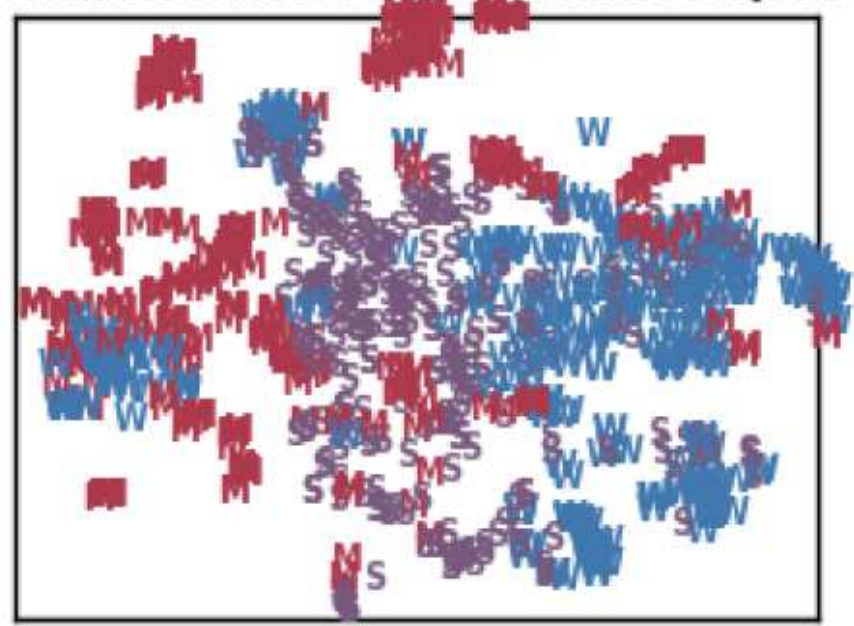

Figure 17

t-SNE embeddings of multimode feature vectors from different subjects 


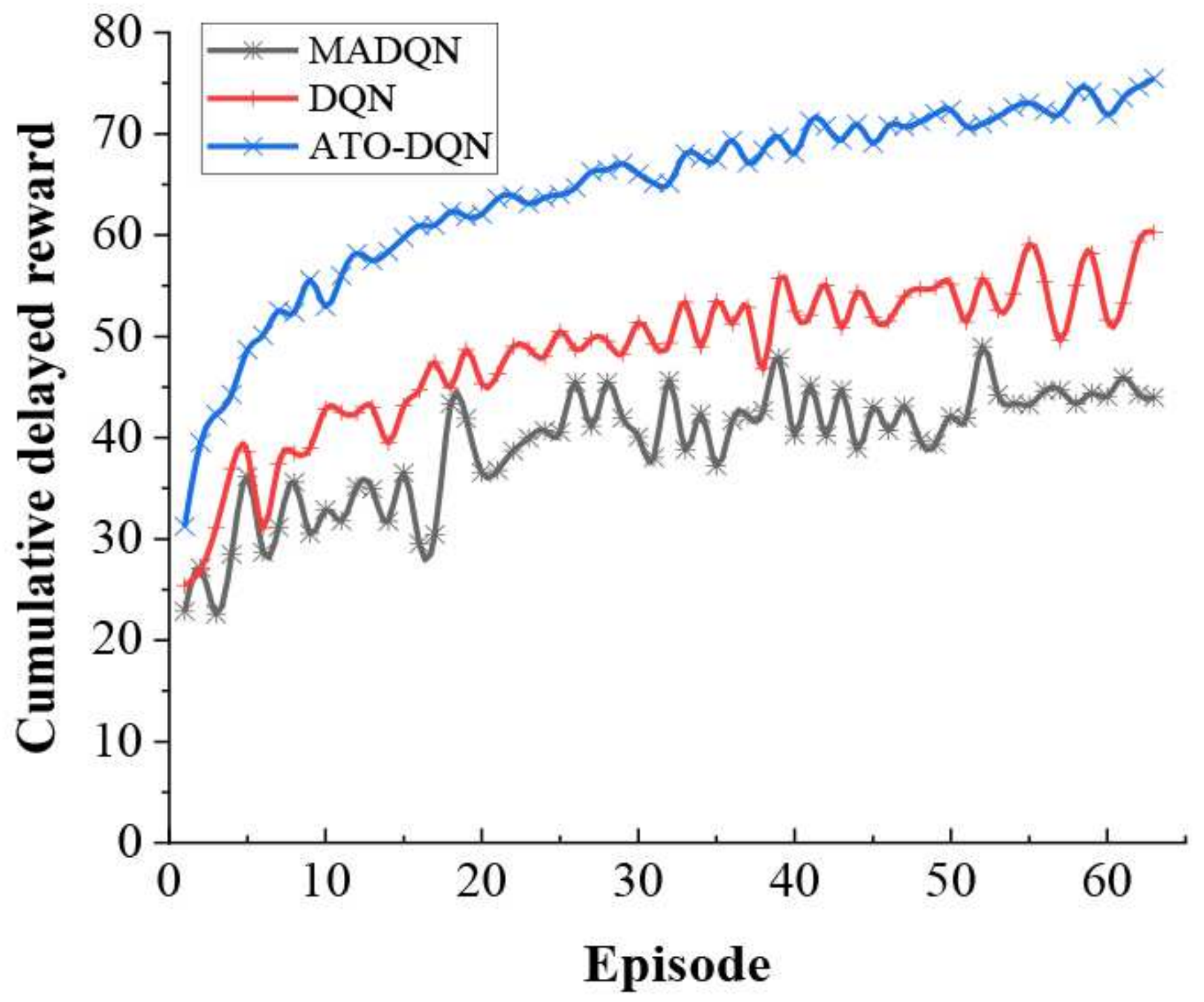

Figure 18

Comparison of cumulative delayed reward for MADQN, traditional DQN and ATO-DQN 


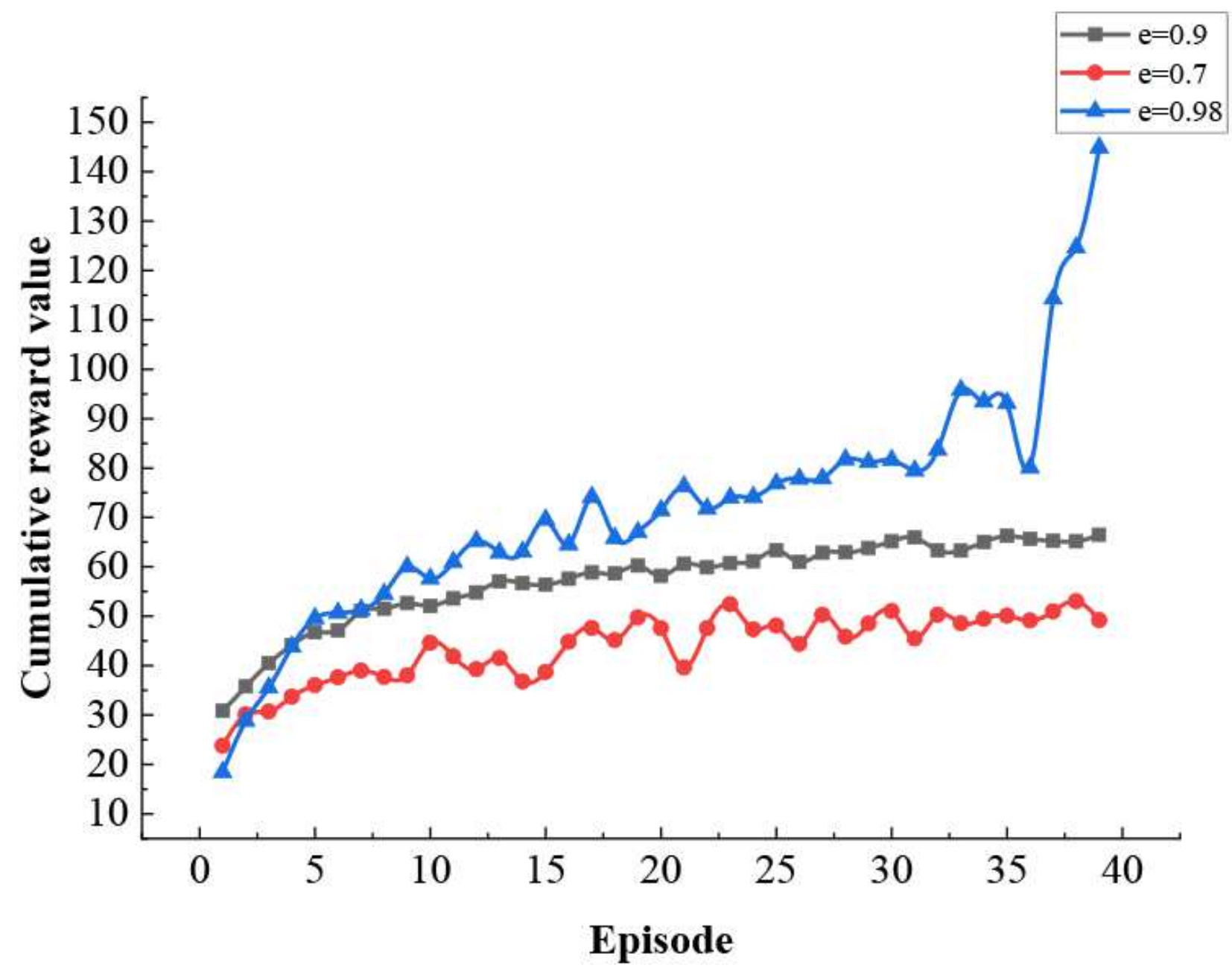

Figure 19

Performance of reward value and convergence rate under different learning rates. When the learning rate $\mathrm{e}$ is 0.7 , the model step length is too short and the convergence speed is too slow; When the learning rate is 0.98 , the convergence speed is greatly improved but can not converge. 


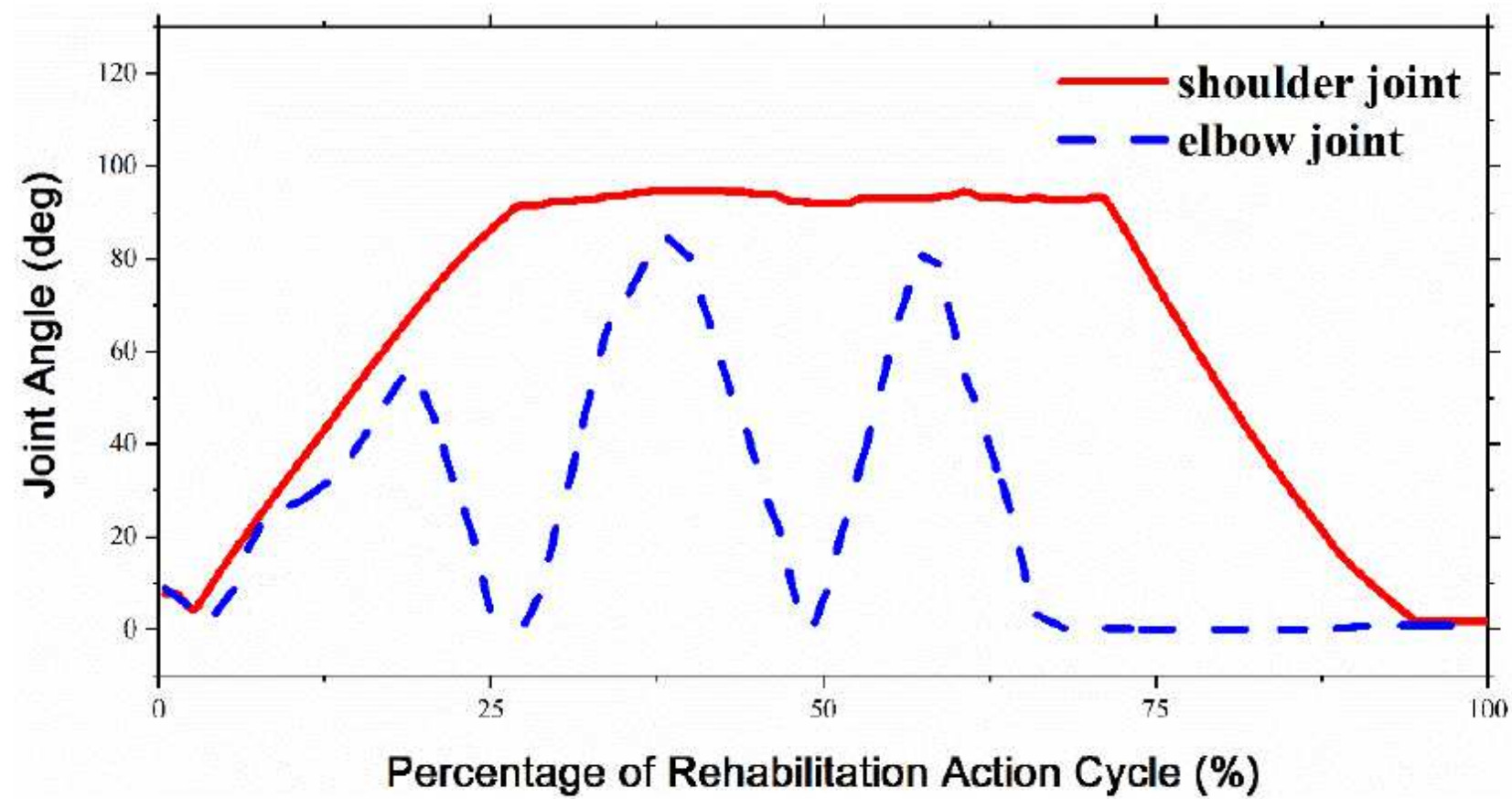

(a). Rehabilitation motion trajectory without ATO-DQN algorithm

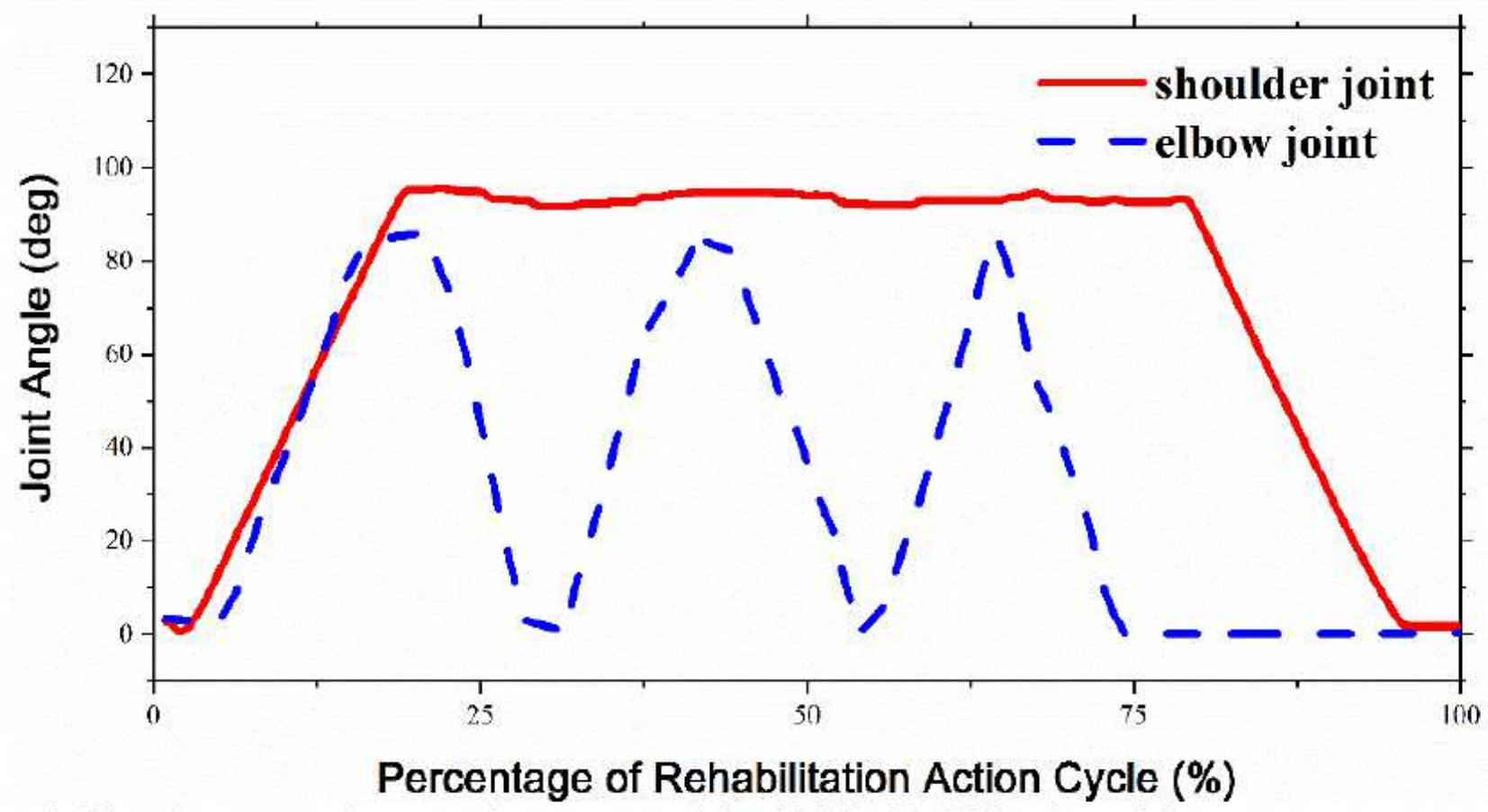

(b). Rehabilitation motion trajectory with ATO-DQN algorithm

Figure 20

Comparison of the trajectories of two states 